Published in final edited form as:

Nature. 2018 January 11; 553(7687): 217-221. doi:10.1038/nature25164.

\title{
Treatment of autosomal dominant hearing loss by in vivo delivery of genome editing agents
}

\author{
Xue Gao ${ }^{1,2,3,11, \# \text {, Yong Tao }}{ }^{4,5,12, \#}$, Veronica Lamas ${ }^{4}$, Mingqian Huang ${ }^{4}$, Wei-Hsi Yeh ${ }^{1,2,3,6}$, \\ Bifeng Pan ${ }^{7}$, Yu-Juan Hu, ${ }^{4,5}$, Johnny H. Hu${ }^{1,2,3}$, David B. Thompson ${ }^{1,2}$, Yilai Shu ${ }^{4,8}$, Yamin \\ $\mathrm{Li}^{9}$, Hongyang Wang ${ }^{4,10}$, Shiming Yang ${ }^{10}$, Qiaobing $\mathrm{Xu}^{9}$, Daniel B. Polley ${ }^{4}$, M. Charles \\ Liberman $^{4}$, Wei-Jia Kong ${ }^{5}$, Jeffrey R. Holt ${ }^{7}$, Zheng-Yi Chen ${ }^{4,}{ }^{,}$, and David R. Liu ${ }^{1,2,3, \dagger,{ }^{*}}$ \\ ${ }^{1}$ Department of Chemistry and Chemical Biology, Harvard University, Cambridge, MA, 02138, \\ USA
}

${ }^{2}$ Howard Hughes Medical Institute, Harvard University, Cambridge, MA, 02138, USA

${ }^{3}$ Broad Institute of MIT and Harvard, Cambridge, MA, 02141, USA

${ }^{4}$ Department of Otolaryngology and Program in Neuroscience, Harvard Medical School and Eaton Peabody Laboratory, Massachusetts Eye and Ear Infirmary, Boston, Massachusetts 02114, USA

${ }^{5}$ Department of Otorhinolaryngology, Union Hospital, Tongji Medical College, Huazhong University of Science and Technology, Wuhan, Hubei 430022, China

${ }^{6}$ Program in Speech and Hearing Bioscience and Technology, Harvard University, Cambridge, Massachusetts 02138, USA

${ }^{7}$ Departments of Otolaryngology and Neurology, F.M. Kirby Neurobiology Center Boston Children's Hospital, and Harvard Medical School, Boston, Massachusetts 02115, USA

${ }^{8}$ Department of Otolaryngology-Head and Neck Surgery, Eye and ENT Hospital, Shanghai Medical College, Fudan University, Shanghai, China

${ }^{9}$ Department of Biomedical Engineering, Tufts University, Medford, MA 02155, USA

Users may view, print, copy, and download text and data-mine the content in such documents, for the purposes of academic research, subject always to the full Conditions of use: http://www.nature.com/authors/editorial_policies/license.html\#termsReprints and permissions information is available at www.nature.com/reprints

"Correspondence should be addressed to Zheng-Yi Chen: Zheng-Yi_Chen@ meei.harvard.edu or David R. Liu: drliu@fas.harvard.edu.

${ }_{11}^{11}$ Current address: Department of Chemical and Biomolecular Engineering, Rice University, Houston, Texas 77005, USA

${ }^{12}$ Current address: Department of Otolaryngology-Head and Neck Surgery, Shanghai Ninth People's Hospital and Ear Institute,

Shanghai Jiaotong University School of Medicine, Shanghai, 200011, China

$\#_{\text {These authors contributed equally to this work }}$

${ }^{\dagger}$ These authors contributed equally to this work

Author contributions

X.G. and Y.T. designed the research, performed genome editing (X.G.) and hearing biology (Y.T.) experiments, analyzed data, and wrote the manuscript. V.L., M.H., W.Y., B.P., Y.H., and H.W. designed experiments, performed hearing biology experiments and analyzed data. D.P., M.C.L., and W.K. designed hearing experiments and analyzed data. Y.S. and S.Y. supported hearing biology experiments. J.H. analyzed GUIDE-seq data. D.T. supported genome editing experiments. Y.L. and Q.X. designed and synthesized lipids. J.H., Z.C., and D.L. designed and supervised the research, and wrote the manuscript. All authors edited the manuscript.

Supplementary Information is available in the online version of the paper.

The authors declare competing financial interests: D.R.L. is a consultant and co-founder of Editas Medicine, Beam Therapeutics, and Pairwise Plants, companies that use genome editing. The co-authors have filed patent applications on aspects of this work.

Correspondence and requests for materials should be addressed to D.R.L. and Z.Y.C. 
${ }^{10}$ Department of Otolaryngology \& Head Neck Surgery, Key Lab of Hearing Impairment Science of Ministry of Education, Key Lab of Hearing Impairment Prevention and Treatment of Beijing City, Chinese PLA Medical School, Beijing, China

\section{Abstract}

Although genetic factors contribute to almost half of all deafness cases, treatment options for genetic deafness are limited ${ }^{1-5}$. We developed a genome editing approach to target a dominantly inherited form of genetic deafness. Here we show that cationic lipid-mediated in vivo delivery of Cas9:guide RNA complexes can ameliorate hearing loss in a mouse model of human genetic deafness. We designed and validated in vitro and in primary fibroblasts genome editing agents that preferentially disrupt the dominant deafness-associated allele in the Tmcl (transmembrane channel-like 1) Beethoven (Bth) mouse model, even though the mutant $B$ th allele differs from the wild-type allele at only a single base pair. Injection of Cas9:guide RNA:lipid complexes targeting the $B t h$ allele into the cochlea of neonatal $B t h /+$ mice substantially reduced progressive hearing loss. We observed higher hair cell survival rates and lower auditory brainstem response (ABR) thresholds in injected ears compared with uninjected ears or ears injected with complexes that target an unrelated gene. Enhanced acoustic reflex responses were observed among injected compared to uninjected $B t h /+$ animals. These findings suggest protein:RNA complex delivery of target gene-disrupting agents in vivo as a potential strategy for the treatment of some autosomal dominant hearing loss diseases.

\section{Keywords}

Genome editing; CRISPR; Cas9; protein delivery; genetic hearing loss

While $\sim 100$ deafness-associated alleles have been identified, few treatments are available to slow or reverse genetic deafness ${ }^{4,5}$. Complementation of wild-type alleles, or silencing dominant negative mutant alleles, have shown promising results in animal models ${ }^{6,7}$. Nonetheless, current approaches face potential challenges including immunogenicity, oncogenicity, and limitations of viral vectors ${ }^{8,9}$.

Cas9-based genome editing agents can mediate targeted gene disruption or repair ${ }^{10-13}$. For applications that seek a one-time, permanent modification of genomic DNA, we and others have shown that the delivery of non-replicable, transient Cas9:single guide RNA (sgRNA) ribonucleotide protein (RNPs) complexes in vivo offer improved DNA specificity and potentially greater safety and applicability ${ }^{14,15}$ compared with methods that introduce DNA expressing these agents. Approximately $20 \%$ of alleles associated with genetic deafness are dominantly inherited ${ }^{1}$. Since Cas9:sgRNA complexes can efficiently disrupt genes through end-joining processes, we sought to design Cas9:sgRNA complexes that selectively disrupt dominant alleles associated with hearing loss.

Many genes linked to genetic hearing loss affect the function of sensory hair cells, which transduce acoustic vibrations into electrical nerve signals. Tmc1 protein is an essential component of mechanotransduction channels in mammalian hair cells ${ }^{16}$. Mutations in TMC1 have been linked to recessive and dominant genetic deafness ${ }^{17}$. A dominant-negative 
missense mutation in $T M C 1$ (p.M418K, c.T1253A) causes reduced single-channel current levels and calcium permeability ${ }^{16}$ and progressive post-lingual sensorineural hearing loss in humans ${ }^{18-20}$. The $B t h /+$ mouse model carries the orthologous missense mutation (p.M412K, c.T1235A) in the mouse $T m c 1$ gene and exhibits progressive auditory response threshold elevation and progressive hair cell loss beginning at one month ${ }^{21}$. Since the orthologous mutations in human and mouse both cause progressive, profound hearing loss, the Bth/t mouse is a promising model for the development of treatment strategies ${ }^{21}$.

We began by developing a genome editing strategy that preferentially disrupts the mouse mutant Tmc1 (Bth) allele. To distinguish the mutant and wild-type alleles, we identified sgRNAs that target $T m c 1$ at sites that include the T1235A mutation and a nearby NGG protospacer-adjacent motif (PAM) sequence required by Streptococcus pyogenes Cas9. We identified three candidate sgRNAs (Tmc1-mut1, Tmc1-mut2, and Tmc1-mut3) that place the Bth mutation at position 11,12, or 15, respectively, of the spacer, counting the PAM as positions 21-23 (Fig. 1a). Mismatches between the sgRNA and genomic DNA that are close to the PAM are poorly tolerated by $\operatorname{Cas} 9^{10}$, increasing the likelihood of selective editing of the Bth mutant allele. A fourth sgRNA, Tmc1-mut4, is a truncated version of Tmc1-mut3 designed to increase genome editing DNA specificity ${ }^{22}$. We evaluated the ability of these four sgRNAs when complexed with Cas9 to cleave either the wild-type Tmc1 or the Bth allele in vitro. All sgRNAs tested preferentially cleaved the Bth allele, with Tmc1-mut3 exhibiting the greatest selectivity (Extended Data Fig 1a and 1b).

We performed lipid-mediated delivery of Cas9:sgRNA RNP complexes into cultured primary fibroblasts derived from wild-type or homozygous Bth/Bth mice to evaluate the allele specificity of genomic DNA modification in mouse cells. We delivered Cas9 complexed with each of the four sgRNAs using Lipofectamine 2000 into both wild-type and Bth/Bth mutant fibroblasts. RNP delivery into these primary fibroblasts was 2- to 4-fold less efficient than with commonly used HEK293T cells (Extended Data Fig. 1c). The highest rate of targeted insertions and deletions (indels) in mutant Bth/Bth fibroblasts (10\%) was observed with Cas9:Tmc1-mut3 RNPs, while lower indel frequencies $(0.74 \%-4.1 \%)$ were observed using the other sgRNAs (Fig. 1b). In contrast, all tested sgRNAs edited the wildtype Tmc1 locus much less efficiently in wild-type fibroblasts (0.066\%-1.6\% indels) (Fig. 1b). Notably, Cas9:Tmc1-mut3 modified the mutant $B$ th allele 23 -fold more efficiently than the wild-type allele (Fig. 1b and Extended Data Fig. 1d). We also prepared three corresponding wild-type Tmc1-targeting sgRNAs (Tmc1-wt1, Tmc1-wt2, and Tmc1-wt3) that lack the T1235A mutation. These sgRNAs edited wild-type fibroblasts on average 10fold more efficiently than Bth/Bth fibroblasts (Extended Data Fig. 1e), confirming that the observed allele selectivities did not arise from the inability of the wild-type Tmc1 allele to be edited.

We tested 17 cationic lipids for their ability to deliver the Cas9:Tmc1-mut3 RNP into Bth/Bth fibroblasts. Several lipids supported substantial modification of the target locus, including RNAiMAX (7.7\%), CRISPRMAX (8.9\%), and Lipofectamine 2000 (12\%) (Extended Data Fig. 2). In contrast, treatment of wild-type fibroblasts with Cas9:Tmc1-mut3 and the same suite of 17 lipids resulted in low $(\leq 0.5 \%)$ indel rates (Extended Data Fig. 2a 
and $2 \mathrm{~b}$ ). These results collectively suggest that the target mutant $T m c 1$ locus can be preferentially disrupted by Cas9:guide RNA complexes.

Exposure of cells to Cas9:sgRNA agents typically results in the modification of both ontarget and off-target loci ${ }^{10,23}$. We used both the GUIDE-seq method ${ }^{23}$ and computational prediction $^{24}$ to identify potential off-target loci that could be modified by Cas9:Tmc1-mut3 exposure. Ten off-target sites containing up to six mismatches in the protospacer region of Tmc1-mut3 sgRNA were identified by GUIDE-seq (Extended Data Fig. 3a). None of these off-target loci are known to be associated with hearing function (Extended Data Table 1a). We measured the indel frequency at each off-target site by HTS in Cas9:Tmc1-mut3 treated Bth/Bth primary mouse fibroblasts following plasmid DNA nucleofection or RNP delivery. Plasmid nucleofection resulted in $0.68 \%-8.1 \%$ indels observed at nine of the ten GUIDE-seqidentified off-target sites (Extended Data Fig. 3b and Table 1a). In contrast, following RNP delivery, modification of only one off-target site (off-T1, 1.2\% indels) was detected (Extended Data Fig. 3b), consistent with our earlier findings that RNP delivery greatly reduces off-target editing compared with DNA delivery ${ }^{15}$. Among the computationally predicted off-target sites ${ }^{24}$, only the two (off- $T 1^{\prime}$ and off-T2') that were also identified as off-targets by GUIDE-seq were observed to undergo modification (Extended Data Table 1b). Together, these results suggest that delivery of Cas9:Tmc1-mut3 RNP complexes into Bth/Bth cells lead to minimal off-target modification, and that phenotypes affecting hearing are unlikely to arise from off-target modification.

To evaluate the ability of the Cas9:Tmc1-mut3 sgRNA complex to target the Bth allele in hair cells in vivo, we complexed Cas9:Tmc1-mut3 sgRNA with Lipofectamine 2000 and injected the resulting mixture into the scala media of neonatal mice by cochleostomy. Neonatal cochlear hair cells express Tmc1 and Tmc2, both of which can enable sensory transduction. To isolate the effect of editing the Bth allele, we injected the Cas9:Tmc1-mut3 sgRNA:lipid complex into Tmc1 Bth/A;Tmc2 $\Delta \Delta$ mice $^{16}$ to avoid transduction current contributions from Tmc 2 and from wild-type Tmc1. We recorded sensory transduction currents from inner hair cells (IHCs) after injection with Cas9:Tmc1-mut3:lipid complex, or with a control targeting an unrelated $g f p$ gene. We observed a significant decline in transduction current amplitudes in Tmc1 Bth/D;Tmc2 $\Delta \Delta$ mice following injection with Cas9:Tmc1-mut3:lipid complexes, consistent with disruption of the $B$ th allele in sensory hair cells in vivo, but not with Cas9:GFP sgRNA:lipid complexes (Fig. 2a and 2b).

In Tmc1 Bth/t;Tmc $2+/+$ mice (abbreviated as $B t h /+$ mice below), IHCs followed by the outer hair cells (OHCs) undergo progressive death ${ }^{21}$. To determine the effect of Cas9:Tmc1mut3 sgRNA on Bth/+ hair cell survival, we injected Cas9:Tmc1-mut3:lipid complex into the scala media of P1 mice and harvested injected and uninjected cochleae after 8 weeks. Uninjected ears exhibited significant loss of IHCs, and partial degeneration of OHCs (Fig. 2c, 2f, and 2g) compared with wild-type C3H ears (Fig. 2e). In injected ears, IHC and OHC survival were substantially enhanced in those regions (Fig. 2d, 2f, and 2g). Stereocilia bundles were observed on surviving IHCs in injected ears, but were absent in uninjected ears in the basal and middle turns (Extended Data Fig. 4a). These results suggest that Cas9:Tmc1-mut3:lipid injection in vivo promotes hair cell survival in $B t h /+$ mice. The strong differences between treated and untreated ears suggests that sporadic disruption of 
Bth may benefit not only edited hair cells, but also surrounding hair cells, consistent with previous findings 25 .

To study the effect of Cas9:Tmc1-mut3:lipid injection on cochlear function in $B t h /+$ mice, we measured auditory brainstem responses (ABRs) that measure the sound-evoked neural output of the cochlea, as well as distortion product otoacoustic emissions (DPOAEs) that measure the amplification provided by $\mathrm{OHCs}^{21}$. In uninjected ears we observed profound attenuation of cochlear neural responses, with ABR thresholds ranging from 70-90 dB at 4 weeks (Fig. 3a and Extended Data Fig. 4b). At 4 weeks, DPOAE threshold elevations in Bth/+ mice were smaller than ABR threshold elevations (Extended Data Fig. 5a), consistent with prior reports that IHCs are more severely affected than OHCs in Bth/t mice. Four weeks after Cas9:Tmc1-mut3:lipid injection, treated Bth/+ ears showed substantially enhanced cochlear function, with lower ABR thresholds relative to uninjected ears at all frequencies below $45 \mathrm{kHz}$ (Fig. 3a). Significant hearing preservation was detected from 8-23 $\mathrm{kHz}$, with average ABR thresholds $15 \mathrm{~dB}$ lower for treated ears than untreated contralateral ears (Fig. 3a; Supplementary Table 1). DPOAE thresholds were slightly elevated in the injected ears, consistent with $\mathrm{OHC}$ damage, perhaps from the injection procedure (Extended Data Fig. 5). We also observed greater ABR Wave 1 amplitudes, and a more normal ABR waveform pattern, in injected ears compared to uninjected controls (Fig. 3b and 3c). Together, these results show that injection of neonatal Bth/+ mice with Cas9:Tmc1mut3:lipid complexes reduces progressive hearing loss.

To test if hearing loss amelioration requires the mutant Bth allele-specific sgRNA, we injected Cas9:Tmc1-wt3:lipid complexes targeting the wild-type Tmc1 allele rather than the Bth mutant allele into P1-2 Bth/+ mice. After four weeks, ABR thresholds in the injected ears were similar to, or worse than, contralateral uninjected ears (Extended Data Fig. 6a; Supplementary Table 1), consistent with the inability of Cas9:Tmc1-wt 3 to efficiently disrupt the Bth allele (Extended Data Fig. 1e), and possible disruption of wild-type Tmc1. Injection of Cas9:sgRNA:lipid complexes targeting an unrelated gene $(g f p)$ did not significantly affect ABR thresholds at most tested frequencies in Bth/+ mice (Extended Data Fig. 6b). To test if preservation of cochlear function requires Cas9 nuclease activity, rather than transcriptional interference from Cas 9 binding to $T m c 1$, we treated $B t h /+$ mice with catalytically inactive dCas $9{ }^{10}$ complexed with Tmc1-mut 1 and observed no evidence of hearing preservation (Extended Data Fig. 5d and 6c; Supplementary Table 1). To evaluate the effects of the treatment on normal mice, we injected Cas9:Tmc1-mut3:lipid into wildtype $\mathrm{C} 3 \mathrm{H}$ mice, the genetic background of the $B t h /+$ mice. We observed similar or slightly elevated ABR thresholds in injected ears relative to uninjected ears 4 weeks after treatment (Extended Data Fig. 6d and 6e), suggesting that Cas9:Tmc1-mut3 does not modify wild-type Tmc1 efficiently enough to substantially affect hearing. Finally, injection of Cas9 and lipid without sgRNA did not improve ABR or DPOAE thresholds (Extended Data Fig. 6f and 6g). Collectively, these results establish that hearing preservation is dependent on sgRNA allele specificity, Cas9 DNA cleavage activity, and the presence of the $B$ th allele. We also characterized cochlear function of $B t h /+$ mice 8 weeks after treatment. Mean ABR thresholds following Cas9:Tmc1-mut3:lipid injection remained lower than uninjected controls from $5.7-23 \mathrm{kHz}$, although the average improvement decreased compared to 4 
weeks post-treatment (Extended Data Fig. 4c and 4d), potentially due to continued progressive hearing loss in the non-edited hair cells.

As a behavioral measure of hearing rescue, we assessed acoustic startle responses 8 weeks after injection. In uninjected $B t h /+$ mice, no startle response was detected following stimuli at $120 \mathrm{~dB}$. In contrast, significant startle responses were detected in Cas9:Tmc1-mut3:lipidinjected $B t h /+$ mice following stimulus at 110 and $120 \mathrm{~dB}$ (Fig. 3d and Extended Data Fig. $4 \mathrm{e})$, demonstrating that hearing preservation upon treatment also preserves an acoustic behavioral reflex.

To evaluate the ability of each of the other Bth-targeting sgRNAs to mediate hearing rescue in vivo, we also injected Tmc1-mut1, Tmc1-mut2, and Tmc1-mut4 complexed with Cas9 into neonatal $B t h /+$ cochleae, and observed varying degrees of enhanced cochlear function (Extended Data Fig. 7). Thus, while Tmc1-mut3 resulted in the most robust hearing preservation, other sgRNAs targeting the mutant $B$ th allele also partially enhanced cochlear function.

To test if RNP delivery of editing agents in adult mouse inner ears supports genome editing in hair cells, we injected Cas9:GFP-targeting sgRNA:lipid complexes into the cochlea of 6week-old Atoh1-GFP mice. Two weeks after injection, GFP fluorescence loss near the injection sites suggested target gene disruption at $25 \pm 2.1 \%$ efficiency (Extended Data Fig. 8 ), comparable to our previous observations of $20 \%$ GFP editing in neonatal hair cells 15 . These results suggest that this approach may be applicable to dominant genetic deafness that manifest with late-onset hearing loss.

To confirm that in vivo treatment of Bth/+ mice with Cas9:Tmc1-mut3 sgRNA disrupted the $B$ th allele, we sequenced DNA from cochlea tissue collected from injected $B t h /+$ and untreated $B t h /+$ mice. After injection at P1, tissues were harvested at P5 and separated into organ of Corti (containing hair cells), spiral ganglion, and spiral ligament samples (Extended Data Fig. 9a and 9b). We estimated the fraction of hair cells in dissected cochlear tissue to be only $\sim 1.5 \%$ of the total cells used for DNA sequencing (Extended Data Fig. 9a and 9b). Nevertheless, we observed unambiguous indels at the Bth locus in cochlear tissue from treated mice (Fig. 4a). The organ of Corti samples contained average Tmc1 editing of $0.92 \%$ of total sequenced DNA, which corresponds to $\sim 1.8 \%$ Bth allele disruption (Fig. $4 \mathrm{a}$ ). We also isolated samples of much smaller numbers of cells (up to a few dozen, mostly hair cells) from treated mice. Decreasing the number of cells entering the genomic DNA amplification and sequencing process increased the observed editing percentage to as high as $10 \% \mathrm{Bth}$ allele disruption, but also elevated background $T m c 1$ indel rates of untreated mice to an average of $0.82 \pm 0.57 \%$ and a maximum of $1.6 \%$, likely reflecting increased noise from processing of minute quantities of genomic DNA. No indel frequencies above that of untreated controls at any of the above-identified off-target sites were observed in Cas9:Tmc1-mut3:lipid-treated tissues (Extended Data Fig. 9c). Together, these observations confirm in vivo editing of the Tmc1 locus from Cas9:Tmc1-mut3:lipid treatment with no detected editing at GUIDE-seq-identified off-target loci. 
Importantly, an analysis of indel-containing $T m c 1$ sequencing reads from treated $B t h /+$ mice allowed us to directly assess the allele specificity of Cas9:Tmc1-mut3 in vivo. Of 11,694 sequencing reads containing indels from four treated organ of Corti samples, 6,118 (52\%) contained an intact nucleotide at Tmc1 position 1235. Of these, 5,736 (94\%) contained modification of the mutant $B$ th allele, while only $382(6 \%)$ contained modification of the wild-type Tmc1 allele (Fig. 4b). Therefore, samples following treatment on average contained 15-fold higher modification of the Bth allele over the wild-type allele (Fig. $4 \mathrm{~b}$ and $4 c)$, demonstrating selective disruption of the $B t h$ allele in $B t h /+$ mice, consistent with observed hearing phenotypes, even though the Bth and wild-type Tmc1 alleles differ only at a single base pair.

This work establishes cationic lipid-mediated Cas9:sgRNA complex delivery in vivo to achieve allele-specific gene disruption in a mouse model of a human genetic disease, resulting in amelioration of a disease phenotype. Our results suggest the potential of such an approach for the treatment of autosomal dominant hearing loss diseases related to hair cell dysfunction, and provide a complementary strategy to other approaches that use antisense oligos (ASO) or RNA interference, 25 . The genome editing strategy developed here may inform the future development of a DNA-free, virus-free, one-time treatment for certain genetic hearing loss disorders.

\section{METHODS}

\section{Primary cell culture}

Wild-type, Bth/+ and Bth/Bth fibroblasts were obtained from P5 pups. Mice were euthanized and cleaned with $70 \%$ ethanol. $1-2 \mathrm{~cm}^{2}$ underarm skin fragments were excised and immerged in cold HBSS (ThermoFisher). Subcutaneous fat was removed by forceps. Skin fragments were cut to $\sim 1 \mathrm{~mm}^{2}$ pieces with a 25G 5/8" Syringe (1180125058, Covidien). Tissues were digested with $0.5 \mathrm{mg} / \mathrm{mL}$ Liberase DL (Sigma 5401160001) at $37^{\circ} \mathrm{C}$ for 1 hour with occasional pipetting up and down to break cell clumps. Warm culture medium (1:1 DMEM:F12 media (ThermoFisher) with 15\% FBS (ThermoFisher) and 100 $\mathrm{U} / \mathrm{mL}$ penicillin+streptomycin (ThermoFisher) was added to stop the enzyme digestion. The solution was filtered with a 70- $\mu \mathrm{m}$ cell strainer (Falcon) and centrifuged at $200 \mathrm{~g}$ for $5 \mathrm{~min}$. The pellet was resuspended in culture medium and transferred to a $25-\mathrm{mL}$ culture flask, then incubated at $37^{\circ} \mathrm{C}, 5 \% \mathrm{CO}_{2}, 3 \% \mathrm{O}_{2}$. Fibroblasts were cultured for $2 \sim 3$ days to reach $\sim 90 \%$ confluence, then passaged in 100-mL flasks in DMEM plus GlutaMax (ThermoFisher) supplemented with $10 \%(\mathrm{v} / \mathrm{v})$ fetal bovine serum (FBS) at $37^{\circ} \mathrm{C}$ with $5 \% \mathrm{CO}_{2}$.

\section{Delivery of proteins complexed with cationic lipids in mouse fibroblasts}

Cultured fibroblast cells were plated in 24-well format (500 $\mu \mathrm{L}$ well volume) in Dulbecco's Modified Eagle's Media plus GlutaMAX (DMEM, Life Technologies) with 10\% FBS (no antibiotics) at a cell density sufficient to reach $\sim 80 \%$ confluence at the time of usage.

Purified sgRNA was incubated with Cas9 protein for 5 min before complexing with cationic lipid ${ }^{15,26}$. Delivery of Cas9:sgRNA was performed by combining $100 \mathrm{nM}$ RNP complex with $3 \mu \mathrm{L}$ cationic lipid in $50 \mu \mathrm{L}$ OPTIMEM media (Life Technologies) according to the manufacturer's protocol for DNA plasmid transfection. The above mixture containing 
cationic lipid and RNP was then added to cells. All complexing steps were performed at room temperature. Cells were harvested and genomic DNA was extracted for sequencing $\sim 96 \mathrm{~h}$ after treatment.

\section{GUIDE-seq and data analysis}

Mouse fibroblasts were transfected using 1,000 ng Cas9 plasmid (pCas9), 300 ng sgRNA plasmid (pTmc1-mut3 sgRNA), and 50 pmol GUIDE-seq double-stranded oligodeoxynucleotides (dsODN) using a LONZA 4D-Nucleofector. Transfection programs were optimized following manufacturer's instructions (CA158 and CA189, P2 Primary Cell 4D-Nucleofector ${ }^{\circledR} \mathrm{X}$ Kit). $400 \mathrm{ng}$ of pmaxGFP Control Vector (LONZA) was added to the nucleofection solution to assess nucleofection efficiencies in primary cells. Media was replaced $\sim 16 \mathrm{~h}$ after nucleofection and cells were harvested for genomic DNA extraction after $\sim 96$ h. For GUIDE-seq off-target DNA cleavage analysis, pCas9, pTmc1-mut3 sgRNA, pmaxGFP, and dsODN were nucleofected into $B t h /+$ heterozygous mouse primary fibroblasts. A sample nucleofected with dsODN only served as a negative control. $\sim 400 \mathrm{ng}$ of genomic DNA for each sample was sheared acoustically using a Covaris m220 sonicator to an average length of $500 \mathrm{bp}$ in $130 \mu \mathrm{L}$ TE buffer. Each sample was sequenced on an Illumina Miseq following previously described protocols ${ }^{23}$. Reads were consolidated first by their Illumina indexes and then by the 8-nt molecular index that defines a single pre-PCR template fragment. The consolidated reads were mapped to the mouse reference genome (GRCm38) using BWA-MEM. Off-target sites were identified by first mapping the start position of the amplified sequences using a 10-bp sliding window, then retrieving the reference sequence around the site. Given the size of some of the deletions, the number of base pairs used as the flanking sequence was increased to $100 \mathrm{bp}$. The retrieved sequences were aligned to the Cas9 target sequence using a Smith-Waterman local-alignment algorithm. The negative control sample treated with the dsODN but no Cas9 or sgRNA was used to assess background.

\section{High-throughput DNA sequencing of genomic DNA samples}

Treated cells or tissues were harvested after four days and genomic DNA was isolated using the Agencourt DNAdvance Genomic DNA Isolation Kit (Beckman Coulter) according to the manufacturer's instructions. On-target and off-target genomic regions of interest were amplified by PCR with flanking HTS primer pairs listed in the Supplementary Sequences. PCR amplification was carried out with Phusion high-fidelity DNA polymerase (ThermoFisher) according to the manufacturer's instructions using 100 ng of genomic DNA as a template. PCR cycle numbers were chosen to ensure the reaction was stopped during the log-linear range of amplification. PCR products were purified using RapidTips (Diffinity Genomics). Purified DNA was amplified by PCR with primers containing sequencing adaptors. The products were purified by gel electrophoresis and quantified using the Quant-iT ${ }^{\mathrm{TM}}$ PicoGreen dsDNA Assay Kit (ThermoFisher) and KAPA Library Quantification Kit-Illumina (KAPA Biosystems). Samples were sequenced on an Illumina MiSeq as previously described ${ }^{27}$.

Sequencing reads were demultiplexed using MiSeq Reporter (Illumina), and individual FASTQ files were analyzed with a custom Matlab script (Supplementary Note). Each read 
was pairwise aligned to the appropriate reference sequence using the Smith-Waterman algorithm. Base calls with a Q-score below 31 were excluded from calculating editing frequencies. Sequencing reads were scanned for exact matches to two 10-bp sequences that flank both sides of a window in which indels might occur. If no exact matches were located, the read was excluded from analysis. If the length of this indel window exactly matched the reference sequence the read was classified as not containing an indel. If the indel window was one or more bases longer or shorter than the reference sequence, then the sequencing read was classified as an insertion or deletion, respectively.

\section{General in vivo experiments}

All in vivo experiments met NIH guidelines for the care and use of laboratory animals and were approved by the Massachusetts Eye \& Ear Infirmary IACUC committee. Isogenic heterozygous $T m c 1 B t h /+$ mice maintained on a $\mathrm{C} 3 \mathrm{HeB} / \mathrm{FeJ}(\mathrm{C} 3 \mathrm{H})$ background were obtained as a gift from Dr. Andrew Griffith ${ }^{21}$, and inbred with wild-type $\mathrm{C} 3 \mathrm{H}$ mice obtained from Jackson Laboratory. Crossbred homozygous C3H-Tmc1 Bth/Bth were caged with C3H mice to generate heterozygous $T m c 1 B t h /+$ mice. All mice were genotyped by Transnetyx (Cordova, TN). For mechanotransduction experiments, two genotypes of Tmc mutant mice were used: $T m c 1$ Bth/Bth;Tmc2 $\Delta \Delta$ and $T m c 1 \Delta \Delta ; T m c 2 \Delta \Delta$ as previously reported ${ }^{16}$.

\section{Microinjection into the inner ear of neonatal mice}

A total of $106 \mathrm{Tmc} 1 \mathrm{Bth} /+$ or $\mathrm{C} 3 \mathrm{H}$ mice (P0-2) of either sex were used for injections. The mice were randomly assigned to the different experimental groups. The final $25 \%$ of the experiments were performed in a double-blinded manner. At least 5 mice were injected in each group. All surgical procedures were done in a clean, dedicated space. Instruments were thoroughly cleaned with $70 \%$ ethanol and autoclaved prior to surgery. Fresh Cas 9 and sgRNA were mixed before injection at a final concentration of $25 \mu \mathrm{M} .1 \mu \mathrm{L}$ Lipofectamine 2000 was mixed with $1 \mu \mathrm{L}$ Cas9:sgRNA RNP and incubated for $20 \mathrm{~min}$ at room temperature. Mice were anesthetized by hyperthermia on ice. Cochleostomy was performed by preauricular incision to expose the cochlear bulla. Anatomic landmarks included the stapedial artery and tympanic ring, which were identified before injection. Glass micropipettes (4878, WPI) were pulled with a micropipette puller (PP83, Narishige) to a final OD of $\sim 10 \mu \mathrm{m}$. Needles held by a Nanoliter 2000 micromanipulator (WPI) were used to manually deliver the Cas9:sgRNA:lipid complexes into the scala media, which allows access to inner ear cells. The injection sites were base, middle, and apex-middle turn of the cochlea. The volume for each injection was $0.3 \mu \mathrm{L}$ with a total volume of $0.9 \mu \mathrm{L}$ per cochlea. The release rate was $69 \mathrm{~nL} / \mathrm{min}$, controlled by MICRO4 microinjection controller (WPI).

\section{Microinjection into adult inner ear by canalostomy}

Three 6-week-old Atoh1-GFP mice ${ }^{28}$ were injected with Cas9:GFP-targeting sgRNA:lipid complex, with the same concentration and volume for each component as used in injection into neonatal inner ear. Mice were anesthetized by intraperitoneal injection of combination of xylazine $(10 \mathrm{mg} / \mathrm{kg})$ and ketamine $(100 \mathrm{mg} / \mathrm{kg})$. The right post-auricular region was exposed by shaving and disinfected by $10 \%$ povidone iodine. For canalostomy, a 10-mm postauricular incision was made under the operating microscope, and the right pinna and the 
sternocleidomastoid muscle were extracted to expose the posterior semicircular canal (PSCC) that was located in the margin of temporal bone. We used a Bonn micro probe (Fine Science Tools, Foster City, CA) to drill a small hole on the PSCC, then left it open for a few minutes until no obvious perilymph leakage was observed. The tip of the polyimide tube (ID 0.0039 inches, OD 0.0049 inches, Microlumen) was inserted into the PSCC toward the ampulla. The hole was sealed with tissue adhesive (3M Vetbond, St. Paul, MN), and the lack of fluid leakage indicated the tightness of the sealing. The tubing was cut after injection, with approximately $5 \mathrm{~mm}$ of tubing left connected to the PSCC and sealed with tissue adhesive. The volume for each injection was $1 \mu \mathrm{L}$ per cochlea. The release rate was $169 \mathrm{~nL} /$ min, controlled by MICRO4 microinjection controller (WPI). The skin was closed with 5-0 nylon suture (Ethicon Inc., Somerville, NJ). The total surgery time was approximately 20 min, including a 6-min injection period.

\section{Acoustic testing}

Auditory brainstem response (ABR) and distortion product otoacoustic emissions (DPOAE) recording were conducted as described previously ${ }^{29}$ at $32{ }^{\circ} \mathrm{C}$ in a soundproof chamber. Mice of either sex were anesthetized with xylazine $(10 \mathrm{mg} / \mathrm{kg}$, i.p.) and ketamine $(100 \mathrm{mg} / \mathrm{kg}$, i.p.). Acoustic stimuli were delivered through a custom acoustic assembly consisting of two miniature dynamic electrostatic earphones (CDMG15008-03A, CUI) to generate primary tones and a miniature microphone (FG-23329-PO7, Knowles) to record ear-canal sound pressure near the eardrum. Custom LabVIEW software controlling National Instruments 24bit soundcards (6052E) generated all ABR/DPOAE stimuli and recorded all responses.

For ABR measurements, needle electrodes were inserted at the vertex and ventral edge of the pinna, with a ground reference near the tail. ABR potentials were evoked with $5 \mathrm{~ms}$ tone pips ( $0.5 \mathrm{~ms}$ rise-fall with a $\cos 2$ onset, delivered at $35 / \mathrm{s})$. The response was amplified 10,000-fold, filtered (100 Hz-3 kHz passband), digitized, and averaged (1024 responses) at each SPL. The sound level was raised in $5 \mathrm{~dB}$ steps from $30 \mathrm{~dB}$ below threshold up to $90 \mathrm{~dB}$ $\mathrm{SPL}$ at frequencies from $5.66-45.24 \mathrm{kHz}$ (in half-octave steps). Following visual inspection of stacked waveforms, "threshold" was defined as the lowest SPL level at which any wave could be detected. In general, thresholds were defined by three independent observers. Wave 1 amplitude was defined as the difference between the average of the 1-ms pre-stimulus baseline and the Wave 1 peak (P1), after additional high-pass filtering to remove lowfrequency baseline shifts.

For DPOAE measurements, the cubic distortion product was measured in response to primaries $\mathrm{f} 1$ and $\mathrm{f} 2$. The primary tones were set so that the frequency ratio (f2/f1) was 1.2 and so that the $\mathrm{f} 2$ level was $10 \mathrm{~dB}$ below the $\mathrm{f} 1$ level. For each $\mathrm{f} 2 / \mathrm{f} 1$ primary pair, primaries were swept in $5 \mathrm{~dB}$ steps from $20 \mathrm{~dB}$ SPL to $80 \mathrm{~dB}$ SPL (for f2). At each level, the amplitude of the DPOAE at 2f1-f2 was extracted from the averaged spectra, along with the noise floor. Threshold was computed by interpolation as the $\mathrm{f} 2$ level required to produce a DPOAE at $5 \mathrm{~dB}$ SPL. 


\section{Acoustic startle reflex}

Mice were placed into a small, acoustically transparent cage resting atop a piezoelectric force plate in a sound attenuated booth. Acoustic stimuli and amplified force plate signals were encoded by a digital signal processor (Tucker-Davis Technologies, RX6) using LabView scripts (National Instruments). Mice were placed in silence for 2 min and $60 \mathrm{~dB}$ broadband white noise for $5 \mathrm{~min}$ to acclimate to the test environment before real measurements. Broadband white noise was presented at a background level of $60 \mathrm{~dB}$ SPL throughout the experiment and a $16-\mathrm{kHz}$ tone was presented at randomized intervals from an overhead speaker ( $80 \mathrm{~dB}$ to $120 \mathrm{~dB}$ SPL, $20 \mathrm{msec}$ duration with $0 \mathrm{msec}$ onset and offset ramps). Ten repetitions were recorded for each of the intensities per test subject. Startle response amplitude was measured as the root mean square (RMS) voltage of the force plate signal shortly after sound presentation.

\section{Immunohistochemistry and histology}

Injected and non-injected cochleae were harvested after animals were sacrificed by $\mathrm{CO}_{2}$ inhalation. Temporal bones were fixed in $4 \%$ paraformaldehyde at $4{ }^{\circ} \mathrm{C}$ overnight, then decalcified in $120 \mathrm{mM}$ EDTA at least 1 week. The cochleae were dissected in pieces from the decalcified tissue for whole-mount immunofluorescence. Tissues were infiltrated with $0.3 \%$ Triton X-100 and blocked with $8 \%$ donkey serum for $1 \mathrm{~h}$ before applying the first antibody. 1:500 rabbit anti-MYO7A (\#25-6790, Proteus BioSciences), 1:750 chicken antiGFP (ab13970, Abcam) and 1:350 goat anti-SOX2 (sc-17320, Santa Cruz Biotechnology) were used at room temperature overnight. The second antibody was incubated for $1 \mathrm{~h}$ after three rinses with PBS rinses. All Alexafluor secondary antibodies were from Invitrogen: donkey anti-rabbit Alex488 (A21206) or Alex 594 (A21207), donkey anti goat Alex594 (A11058) or Alexa-488-phalloidin (A12379) and goat anti-chicken Alex488 (A-11039) were used as a 1:500 dilution. Specimens were mounted in ProLong Gold Antifade Mountant medium (P36930, Life Technologies). Confocal images were taken with a Leica TCS SP5 microscope using a $20 \times$ or $63 \times$ glycerin-immersion lens, with or without digital zoom. For IHC and OHC counting, we acquired z-stacks by maximum intensity projections of z-stacks for each segments by imageJ (NIH image), and composite images showing the whole cochlea were constructed in Adobe Photoshop CS3 to show the whole turn of cochlea. A frequency map was constructed for each case by measuring the spiral extent of all the dissected cochlear pieces and converting cochlear location to frequency using a plug-in of ImageJ (http://www.masseyeandear.org/research/ent/eaton-peabody/epl-histology-resources/ imagej-plugin-for-cochlear-frequency-mapping-in-whole-mounts/). MYO7A positive IHCs and $\mathrm{OHC}$ were counted in the cochlear regions that respond to different sound frequencies, and any segments containing dissection-related damage were omitted from further analysis.

\section{Hair cell transduction current recording}

Tmc1 Bth/Bth;Tmc2 $\Delta / \Delta$ and Tmc1 $\Delta \Delta, T m c 2 \Delta \Delta$ littermates were injected with $0.9 \mu \mathrm{L}$ Cas9:Tmc1-mut3 sgRNA:Lipofectamine 2000 or Cas9:GFP sgRNA: Wild-type C57B/L6 mice were injected with $0.9 \mu \mathrm{L}$ Cas9:Tmc1-wt3 sgRNA:Lipofectamine 2000 Lipofectamine 2000 at P0-P1 via the cochleostomy approach. Cochleae were harvested at P5-P6 and cultured in $\operatorname{MEM}(1 \mathrm{X})+$ GlutaMAX ${ }^{\mathrm{TM}}-\mathrm{I}$ medium with $1 \% \mathrm{FBS}$ at $37{ }^{\circ} \mathrm{C}, 5 \% \mathrm{CO}_{2}$ for up to 
15 days. For recording, the organs of Corti were bathed in standard artificial perilymph containing $137 \mathrm{mM} \mathrm{NaCl}, 0.7 \mathrm{mM} \mathrm{NaH}_{2} \mathrm{PO}_{4}, 5.8 \mathrm{mM} \mathrm{KCl}, 1.3 \mathrm{mM} \mathrm{CaCl}_{2}, 0.9 \mathrm{mM} \mathrm{MgCl}_{2}$, $10 \mathrm{mM}$ HEPES, and $5.6 \mathrm{mM}$ D-glucose. Vitamins (1:50) and amino acids (1:100) were added to the solution from concentrates (Invitrogen, ThermoFisher Scientific), and $\mathrm{NaOH}$ was used to adjust the final $\mathrm{pH}$ to $7.4(\sim 310 \mathrm{mOsm} / \mathrm{kg})$. Recording pipettes $(2-4 \mathrm{M} \Omega)$ were pulled from R6 capillary glass (King Precision Glass) and filled with intracellular solution containing $135 \mathrm{mM} \mathrm{CsCl}, 5 \mathrm{mM}$ HEPES, $5 \mathrm{mM}$ EGTA, $2.5 \mathrm{mM} \mathrm{MgCl} 2,2.5 \mathrm{mM} \mathrm{Na}_{2}$-ATP, and $0.1 \mathrm{mM} \mathrm{CaCl}_{2} ; \mathrm{CsOH}$ was used to adjust the final $\mathrm{pH}$ to $7.4(\sim 285 \mathrm{mOsm} / \mathrm{kg})$. Wholecell, tight-seal, voltage-clamp recordings were conducted at $-84 \mathrm{mV}$ at room temperature $\left(22-24{ }^{\circ} \mathrm{C}\right)$ with an Axopatch 200B amplifier (Molecular Devices). Hair bundles were deflected with a stiff glass probe fabricated from capillary glass with a fire polisher (MF-200, World Precision Instruments) for creating a rounded probe tip of $\sim 3-5 \mu \mathrm{m}$ in diameter. Probes were mounted on a PICMA Chip piezo actuator (Physik Instrument, Karlsruhe, Germany) and driven by an LVPZT amplifier (E-500.00, Physik Instrumente, Karlsruhe, Germany). Sensory-transduction currents were recorded from uninjected and Cas9:sgRNA-treated hair cells. The data were filtered at $10 \mathrm{kHz}$ with a low-pass Bessel filter and digitized at $>20 \mathrm{kHz}$ with a 16-bit acquisition board (Digidata 1440A, Molecular Devices) and pClamp 10 software (Molecular Devices).

\section{Inner ear tissue dissection for HTS}

Tmc1 Bth/+ mice were injected with Cas9:sgRNA at P1 as described above. All dissection instruments were thoroughly cleaned with 70\% ethanol and DRNAase Free (D6002, ARgos), then autoclaved prior to dissection. Mice were euthanized at P5. Temporal bones were removed and immersed in clean PBS pH 7.4 (10010001, ThermoFisher) individually. Different forceps were used for each ear. The organ of Corti, spiral ganglion, and spiral ligament from the injected and non-injected ear, and tail tissue were all harvested under microscope from each mouse.

\section{Hair cells isolation for HTS}

Tmc1 Bth/+ mice were injected with Cas9:Tmc1-mut3 sgRNA:Lipofectamine 2000 at P1 and euthanized at P5. Cochleae were dissected and immersed in $1 \mu \mathrm{M}$ FM 1-43FX (PA1-915, ThermoFisher) dissolved in HBSS (ThermoFisher) for $10 \mathrm{sec}$ at room temperature in the dark. Cochleae were rinsed three times with HBSS and placed in $100 \mu \mathrm{L}$ Cell Recovery Solution (354253, Discovery Labware) for $10 \mathrm{~min}$ at $37{ }^{\circ} \mathrm{C}$, then transferred to $100 \mu \mathrm{L}$ TrypleE Express Enzyme (12604013, ThermoFisher). Sensory epithelium were extracted by forceps. After incubation for $10 \mathrm{~min}$ at $37{ }^{\circ} \mathrm{C}$, the tissues were pipetted up and down 30 times. FM 1-43-positive cells were isolated by 1- $\mu \mathrm{L}$ pipette under a microscope (Axiovert 200M, Carl Zeiss), then subjected to whole genome amplification by MALBAC Single Cell WGA Kit (YK001A, Yikon Genomics).

\section{Statistical analysis}

Statistical analyses were performed by two-way ANOVA with Bonferroni corrections for multiple comparisons for ABRs, DPOAEs, and acoustic startle response; and by Student's ttest for hair cell transduction currents using Prism 6.0 (GraphPad). 


\section{Data availability}

High-throughput sequencing data is deposited in the NCBI Sequence Read Archive database under accession code SRP103108.

\section{Code availability}

Labview software for cochlear function testing is available here: http://

www.masseyeandear.org/research/otolaryngology/investigators/laboratories/eaton-peabodylaboratories/epl-engineering-resources. Matlab scripts used to quantify the acoustic startle response are available on request. Indel identification scripts are in the Supplementary Information.

\section{Extended Data}

a

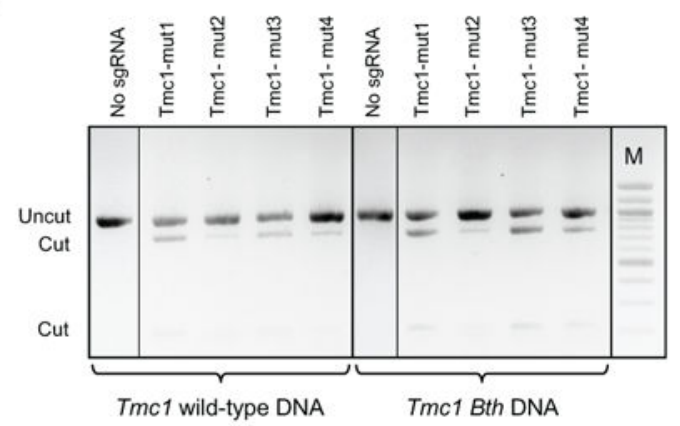

b

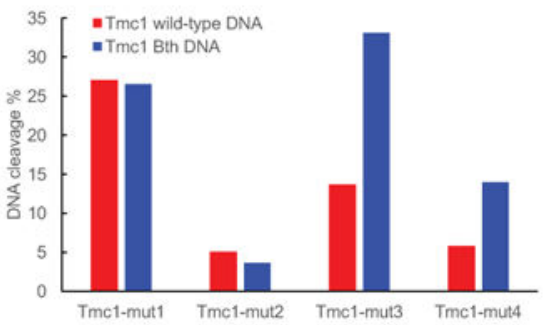

C

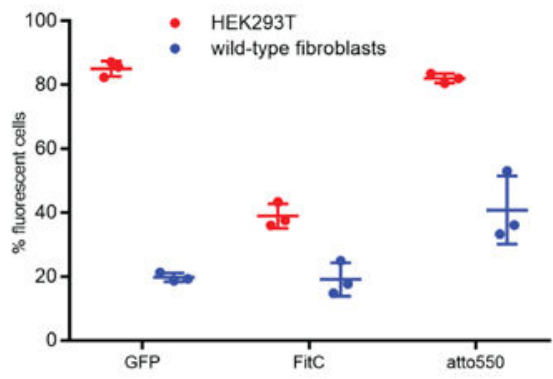

d

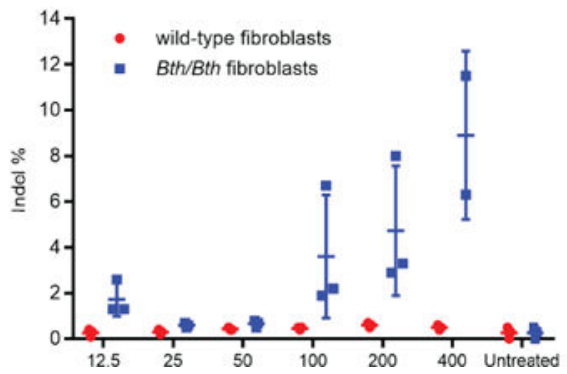

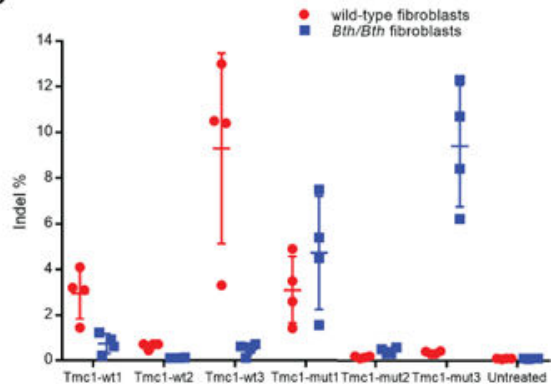

Extended Data Figure 1.

Allele-selective editing of wild-type or Bth mutant Tmc1 in cleavage assays in vitro and in lipid-mediated delivery into primary fibroblasts. (a) In vitro Cas9:sgRNA-mediated Tmc1 DNA cleavage. $100 \mathrm{nM}$ of a 995-bp DNA fragment containing wild-type Tmc1 (lanes 1-5) or Bth mutant Tmc1 (lanes 6-10) was incubated with $300 \mathrm{nM}$ of each of the four Cas9:sgRNAs shown for $15 \mathrm{~min}$ at $37^{\circ} \mathrm{C}$. Expected cleavage products are 774-778 bp and 217-221 bp. $\mathrm{M}=100$-bp ladder; the lower two heavy bands are 500 and 1,000 bp. (b) Quantification of DNA cleavage in (a) by densitometry using imageJ. (c) Transfection efficiency comparison of HEK293T cells and wild-type primary fibroblasts. $50 \mathrm{ng}$ GFP plasmid, 10 nM Cas9:FitC-Tmc1-mut3 sgRNA RNP, or 10 nM Cas9:CrRNA-Tmc1mut3:atto-550-TracrRNA RNP were delivered into HEK293T cells or wild-type primary fibroblasts using $3 \mu \mathrm{L}$ Lipofectamine 2000. For samples with GFP plasmid, the fraction of GFP-positive cells was measured by flow cytometry $24 \mathrm{~h}$ after delivery. For samples with 
Cas9:FitC-Tmc1-mut3 RNP, or Cas9:CrRNA-Tmc1-mut3:atto-550-TracrRNA RNP, media was removed $6 \mathrm{~h}$ after delivery. The cells were trypsinized, washed three times with $500 \mu \mathrm{L}$ PBS containing $20 \mathrm{U} / \mathrm{mL}$ heparin, and subjected to flow cytometry. (d) Wild-type or Bth mutant $T m c 1$ allele editing in primary fibroblasts derived from wild-type or Bth/Bth mice as a function of the dose of Cas9:Tmc1-mut3:lipid complex. 12.5, 25, 50, 100, 200, or $400 \mathrm{nM}$ of Cas9:Tmc1-mut3 were delivered into the primary fibroblasts using Lipofectamine 2000 in DMEM-FBS. (e) Lipid-mediated delivery of Cas9:sgRNA complexes into primary fibroblasts derived from wild-type or Bth/Bth mice. $100 \mathrm{nM}$ of purified Cas9 protein and each wild-type Tmc1-targeting sgRNA (Tmc1-wt1, Tmc1-wt2, or Tmc1-wt3) or Bth mutant-targeting sgRNA (Tmc1-mut1, Tmc1-mut2, or Tmc1-mut3) were delivered into wild-type fibroblasts (red) and Bth/Bth fibroblasts (blue) using Lipofectamine 2000 in DMEM-FBS. Primary fibroblast cells were harvested $96 \mathrm{~h}$ after treatment. Genomic DNA was extracted and indels were detected by HTS. Values and error bars reflect the mean \pm standard deviation of three or more biological replicates. 
a

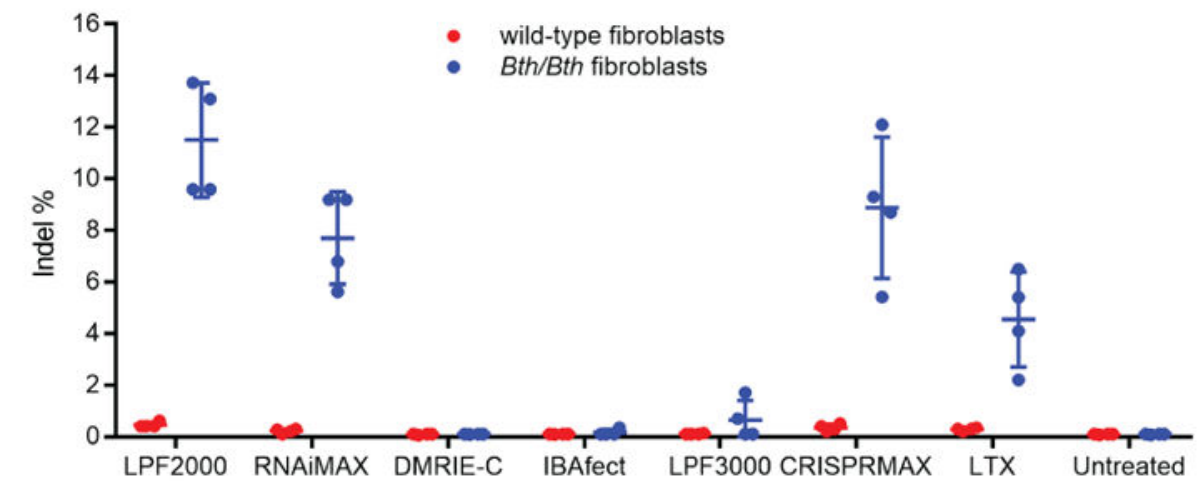

b

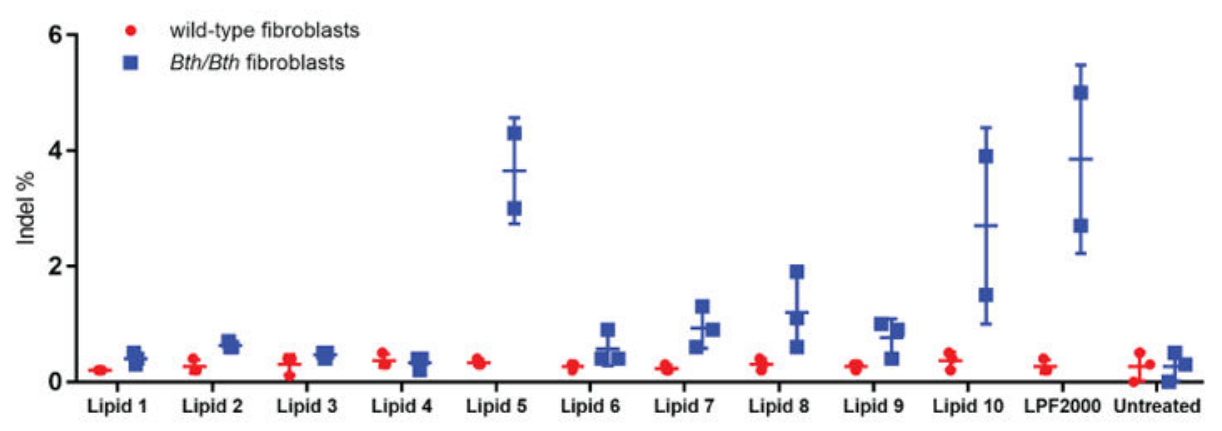

C

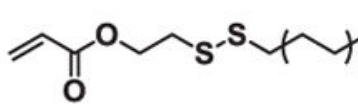

О12B $(x=3)$, O14B $(x=4)$, 016B $(x=5), 018 B(x=6)$

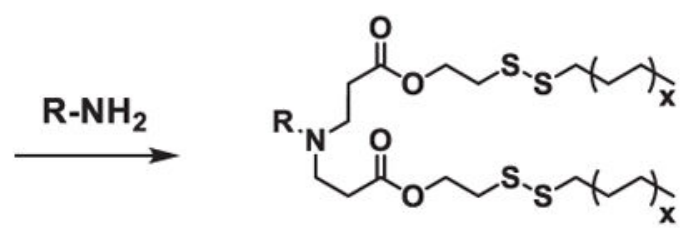

R-012B ( $x=3$ ), R-014B ( $x=4)$, R-016B ( $x=5), R-018 B(x=6)$

d<smiles>CCN(CC)CCN</smiles>

75<smiles>NCCN1CCCC1</smiles>

76<smiles>CN(C)CCCN</smiles>

80<smiles>NCCCN(CCO)CCO</smiles>

87<smiles>CN(CCN)CCN</smiles>

113

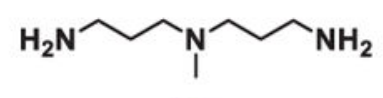

306

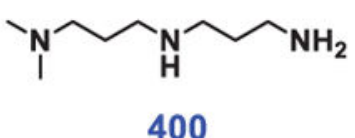

Extended Data Figure 2.

Delivery of Cas9:Tmc1-mut3 sgRNA complexes into primary fibroblasts derived from wildtype or homozygous Bth/Bth mice using (a) seven commercially available lipids. LPF2000 = Lipofectamine 2000; RNAiMAX = Lipofectamine ${ }^{\circledR}$ RNAiMAX; LPF3000 = Lipofectamine 3000; CRISPRMAX = Lipofectamine CRISPRMAX; LTX = Lipofectamine LTX, or (b) ten biodegradable, bioreducible lipids. Lipid $1=75-\mathrm{O} 14 \mathrm{~B}$; Lipid $2=76-\mathrm{O} 14 \mathrm{~B}$; Lipid $3=80$ O18B; Lipid 4 = 87-O16B; Lipid 5 = 113-O18B; Lipid 6 =306-O12B; Lipid 7 = 306-O16B; Lipid $8=306-O 18 B ;$ Lipid $9=400-O 12 B ;$ Lipid 10 =400-O16B. $100 \mathrm{nM}$ purified 
Cas9:Tmc1-mut3 RNP was delivered using $3 \mu \mathrm{L}$ of the cationic lipid shown in DMEM-FBS. Fibroblast cells were harvested $96 \mathrm{~h}$ after treatment, genomic DNA was extracted, and indels were detected by HTS. (c) Synthetic route and chemical structure of lipids. (d) Commercially available amine head groups used in lipid synthesis. Lipids were synthesized as previously described ${ }^{26}$. Values and error bars reflect the mean \pm standard deviation of three or more biological replicates.

a

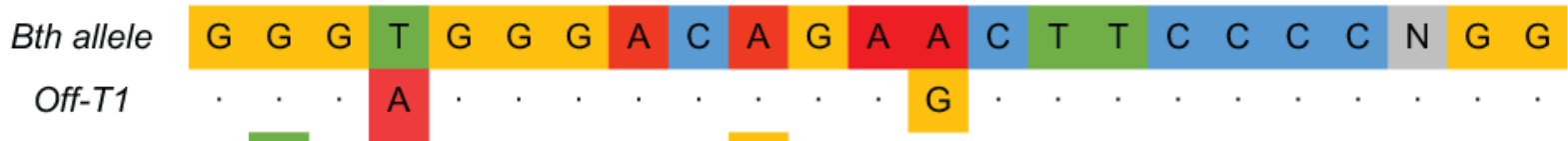

Off-T2 $\cdot \mathrm{T} \cdot \mathrm{A} \cdot \cdot \cdot \cdot \cdot \mathrm{G}$

Off-T3

Off-T4

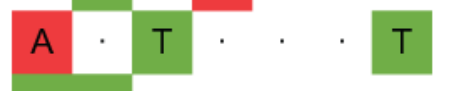

Off-T5

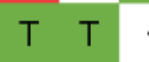

Off-T6

Off-T7
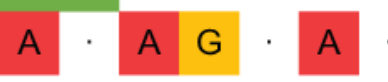

Off-T8

Off-T9

Off-T10
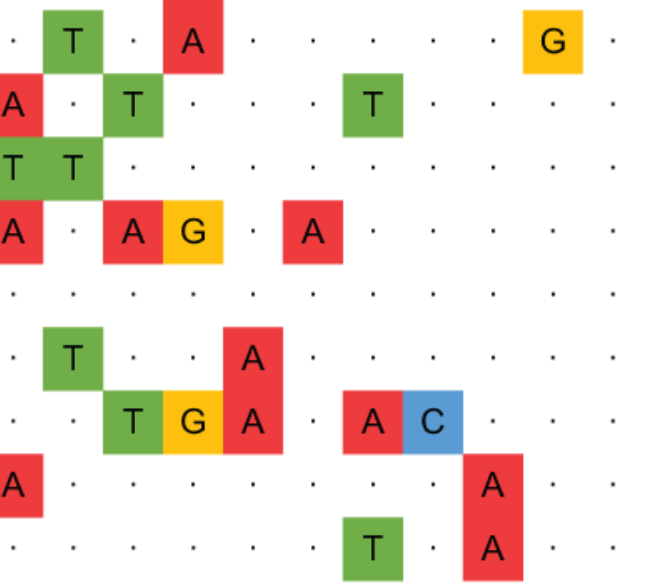

b

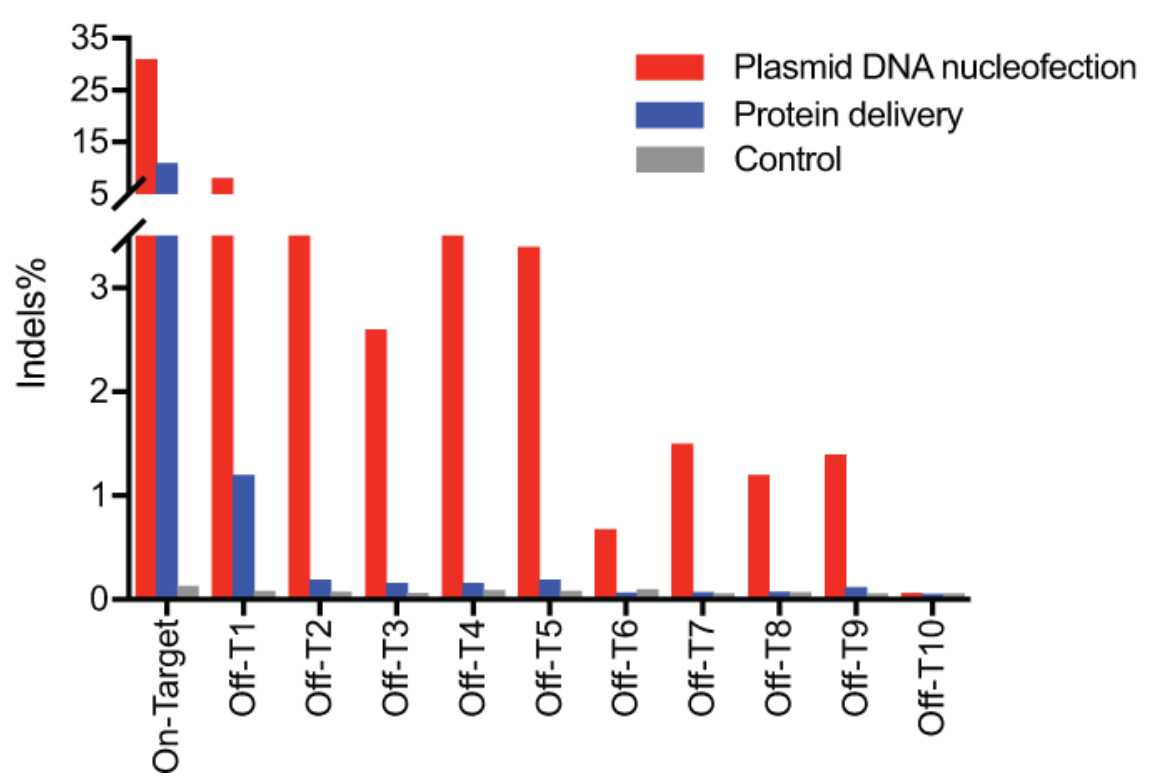

Extended Data Figure 3.

Off-target sites identified by GUIDE-seq after nucleofection of DNA plasmids encoding Cas 9 and Tmc1-mut 3 sgRNA into primary fibroblasts from $B t h /+$ mice. (a) 1,000 ng Cas9 plasmid, 300 ng Tmc1-mut3 sgRNA plasmid, 400 ng pmaxGFP plasmid, and 50 pmol double-stranded oligodeoxynucleotides (dsODN) were nucleofected into $B t h /+$ fibroblasts 
using a LONZA 4D-Nucleofector. Genomic DNA was extracted $96 \mathrm{~h}$ after nucleofection and subjected to GUIDE-seq as previously described ${ }^{25}$. Off- $T 1$ to off-T1O are ten off-target sites detected by GUIDE-seq. Mismatches compared to the on-target site are shown and highlighted in color. The Bth allele targeted by sgRNA Tmc1-mut3 is shown in the top row. (b) Indel frequency at the Tmc1 locus and at each of the off-target loci in Cas9:Tmc1-mut3 treated Bth/Bth primary fibroblasts following plasmid DNA nucleofection or following RNP delivery. For RNP delivery, $100 \mathrm{nM}$ Cas9:Tmc1-mut3 RNP was delivered to the Bth/Bth fibroblasts using $3 \mu \mathrm{L}$ Lipofectamine 2000. Indels were detected by HTS at the Tmc1 ontarget site and at each off-target site. Red: samples nucleofected with DNA plasmids encoding Cas9 and Tmc1-mut3 sgRNA; blue: samples treated with Cas9:Tmc1-mut3 RNPs; grey: control samples nucleofected with unrelated dsDNA only. 
a

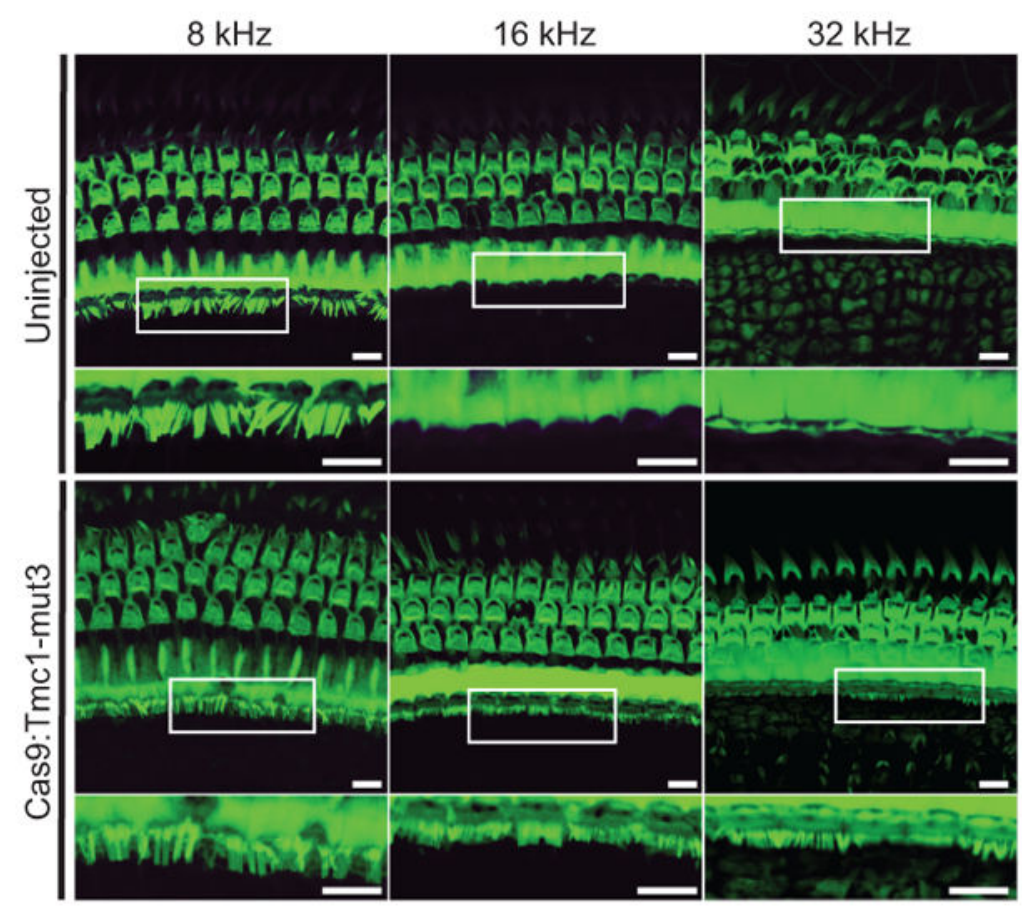

b

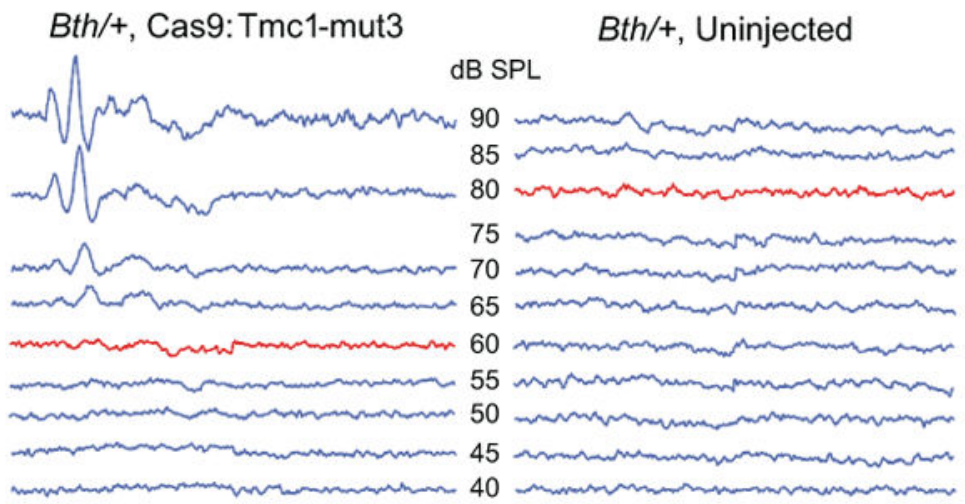

C

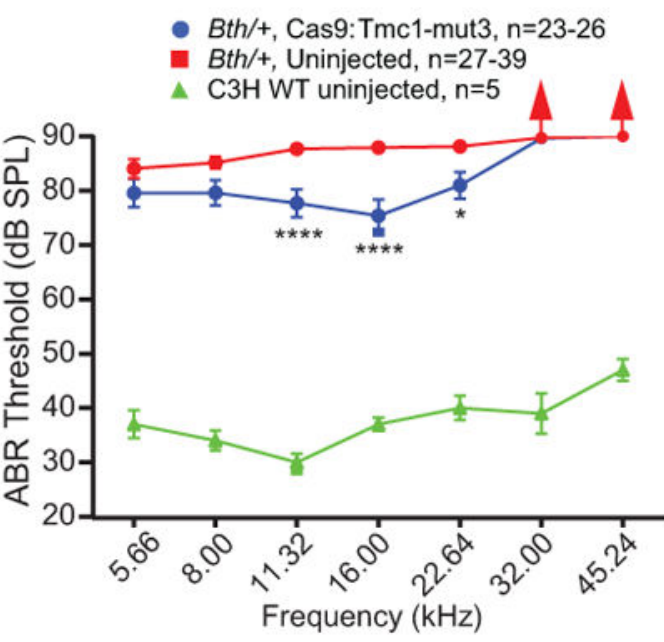

d

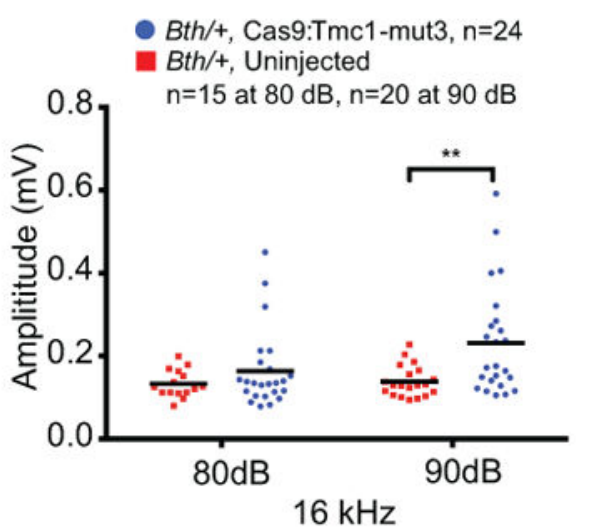

e

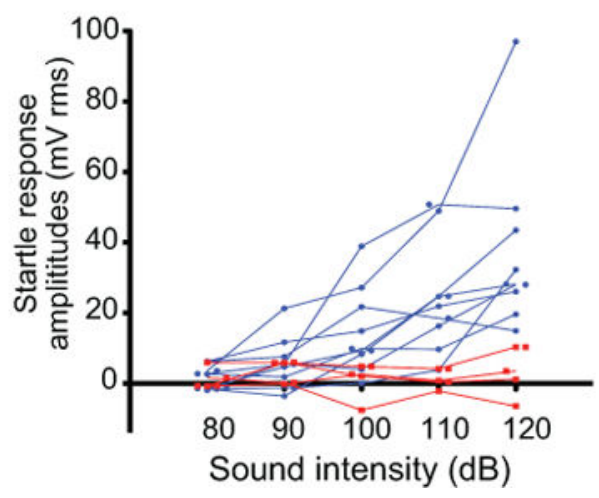

Extended Data Figure 4.

Cas9:Tmc1-mut3:lipid injection reduce hearing loss, improve acoustic startle response, and preserve stereocilia in $B t h /+$ mice. (a) Phalloidin labeling showed the preservation of stereocilia of IHCs in an ear 8 weeks after injection with Cas9:Tmc1-mut3 sgRNA at three frequency locations indicated, whereas the uninjected contralateral inner ear of the same mouse showed severe degeneration of stereocilia at locations corresponding to 16 and 32 $\mathrm{kHz}$. The boxes indicate the stereocilia, which are shown at the bottom of each image at higher magnification. Scale bars: $10 \mu \mathrm{m}$. Similar results were observed in other injected ears that were immunolabeled $(n=5)$. (b) Representative ABR waveforms showing reduced 
threshold (red traces) at $16 \mathrm{kHz}$ in a Cas9:Tmc1-mut3:lipid-injected $B t h /+$ ear (left) compared to an uninjected contralateral ear (right) after 4 weeks. (c) 8 weeks after Cas9:Tmc1-mut3 injection into $B t h /+$ ears (blue), mean ABR thresholds were significantly reduced at three frequencies. Uninjected $B t h /+$ ears (red) showed ABR thresholds $>85 \mathrm{~dB}$ at all frequencies after 8 weeks. ABR thresholds from wild-type $\mathrm{C} 3 \mathrm{H}$ are shown in green. (d) ABR Wave 1 amplitudes following $90 \mathrm{~dB}$ SPL at $16 \mathrm{kHz}$ were greater in injected $B t h /+$ ears than in uninjected ears 8 weeks after treatment. The horizontal bars represent mean values. (e) Startle responses at $16 \mathrm{kHz}$ in individual Cas9:Tmc1-mut3 sgRNA-injected mice (blue) were significantly stronger $(p<0.001)$ than in uninjected mice (red) 8 weeks after treatment. Among the different frequencies assayed, the number of ears tested (n) varies within the range shown (see Supplementary Table 2). Statistical analyses of ABR thresholds, amplitudes, and startle responses were performed by two-way ANOVA with Bonferroni correction for multiple comparisons: ${ }^{*} p<0.05$, ${ }^{* *} p<0.01$, and $* * * * p<0.0001$. Values and error bars reflect mean \pm SEM.

a

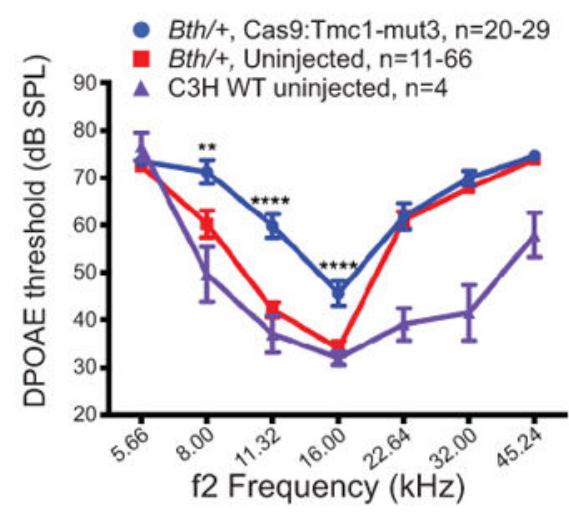

d

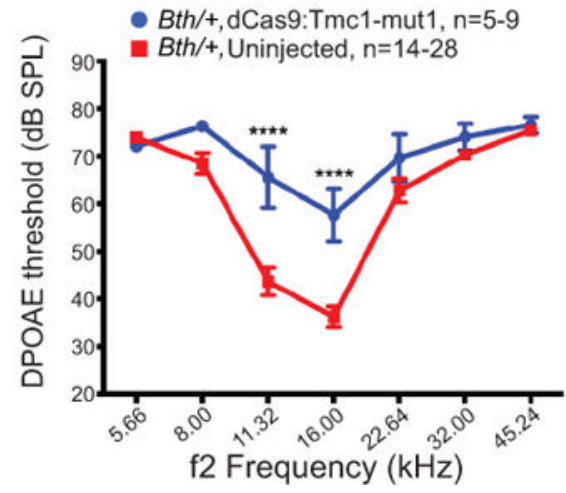

b

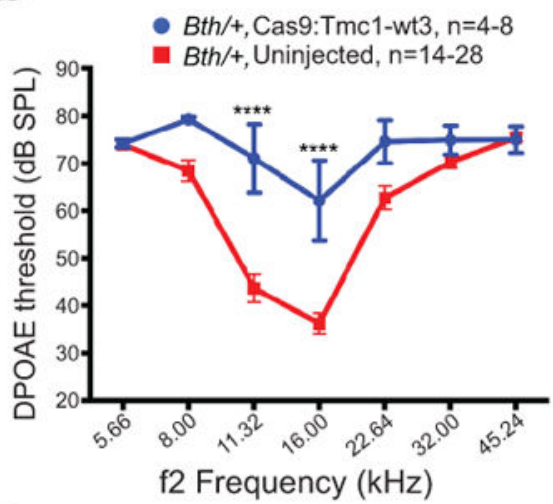

e

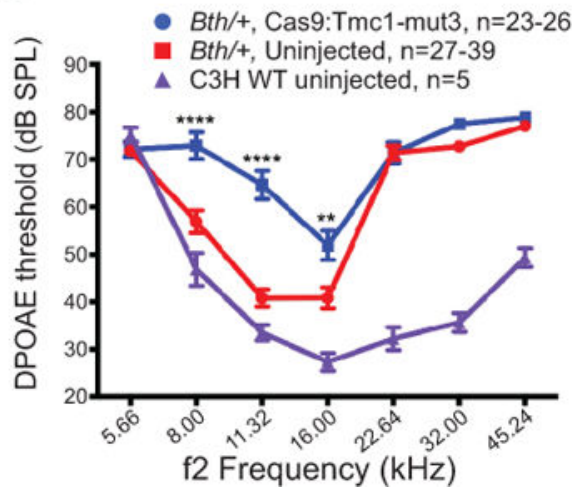

C

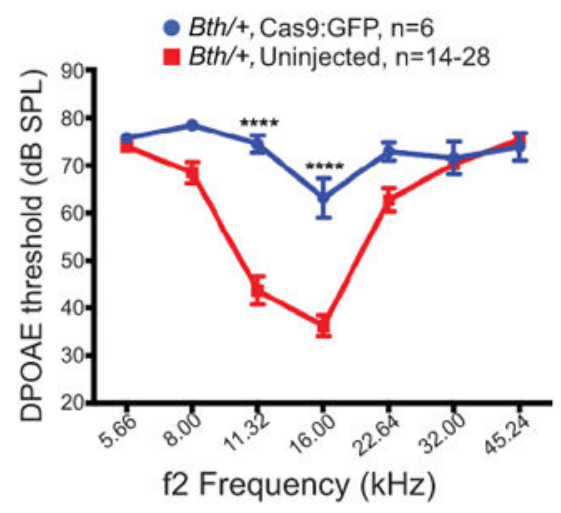

Extended Data Figure 5.

Effect of in vivo injection of Cas9:sgRNA:lipid complexes on DPOAE thresholds. DPOAE thresholds 4 weeks after injection were elevated compared with uninjected ears at three frequencies following treatment with Cas9:Tmc1-mut3 sgRNA (a), and were elevated at two frequencies following treatment with Cas9:Tmc1-wt3 sgRNA, (b) Cas9:GFP sgRNA (c), or dCas9:Tmc1-mut1 sgRNA (d). (e) 8 weeks after Cas9:Tmc1-mut3 sgRNA injection, DPOAE thresholds were elevated at three frequencies in the injected group. Mean DPOAE 
thresholds of untreated wildtype (WT) $\mathrm{C} 3 \mathrm{H}$ mice at 4 weeks (a) or 8 weeks (e) weeks of age are also shown in purple. Statistical analysis of DPOAE thresholds was performed by twoway ANOVA with Bonferroni correction for multiple comparisons: $* * p<0.01$, *** $p<$ 0.001 , and $* * * * p<0.0001$. Values and error bars reflect mean \pm SEM. Among the different frequencies assayed, the number of ears tested (n) varies within the range shown (see Supplementary Table 2). The elevation of DPOAE thresholds despite enhanced hair cell survival (Fig. $2 \mathrm{~d}$ and $2 \mathrm{~g}$ ) suggests that the surviving OHCs may not be fully functional. IHCs can respond to sound and excite auditory nerve fibers in the absence of OHC amplification, although at higher SPLs. Thus, an improvement in ABR thresholds and suprathreshold amplitudes can occur without concomitant DPOAE enhancement if the functional improvements are restricted to the surviving IHCs.

a

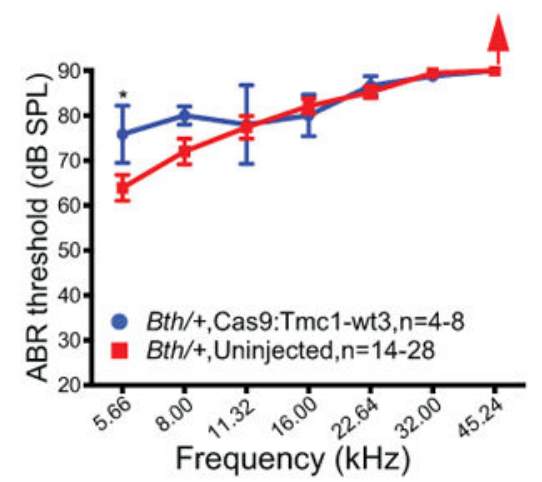

b

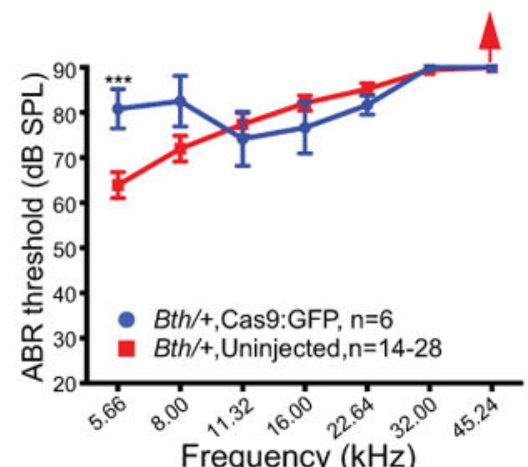

C

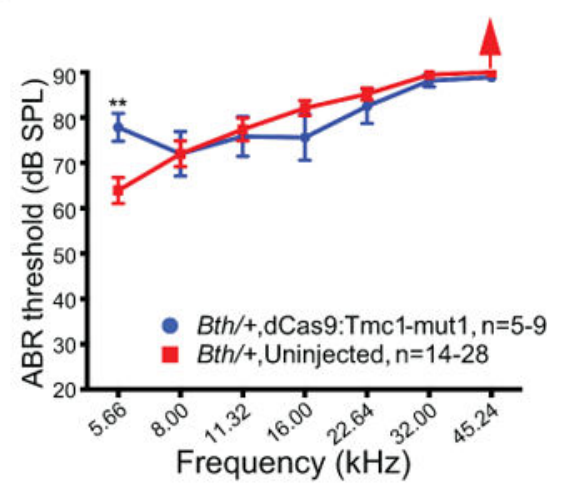

d

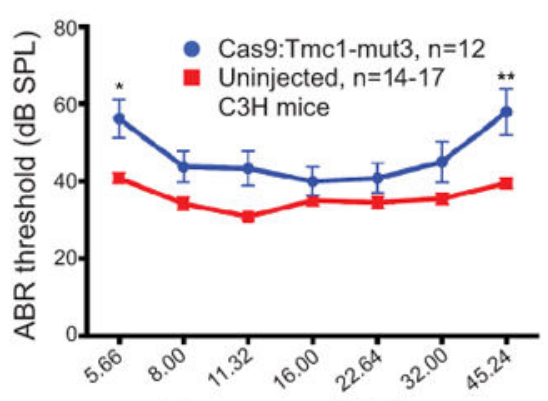

Frequency $(\mathrm{kHz})$

f

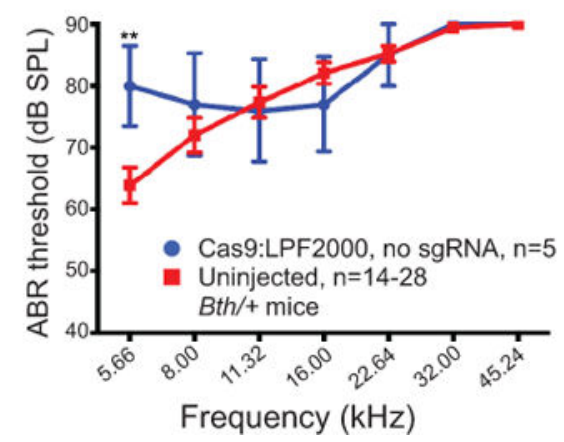

e

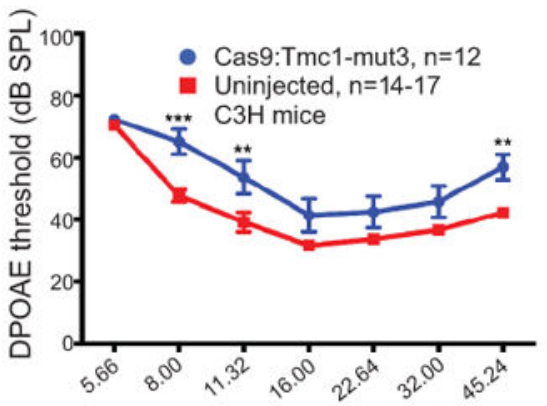

f2 Frequency $(\mathrm{kHz})$

g

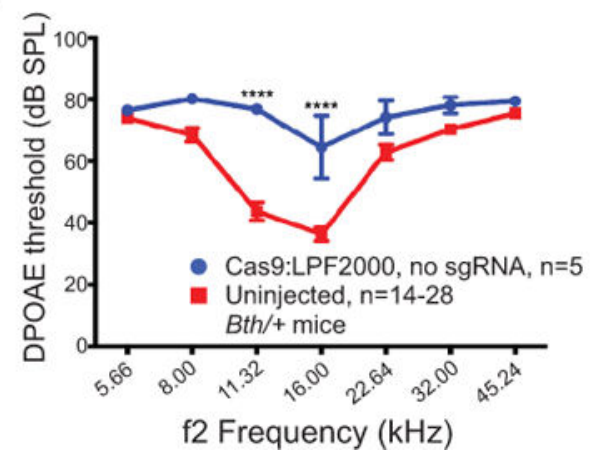


Extended Data Figure 6.

Hearing rescue is dependent on the $B t h$ target specificity of the sgRNA, Cas 9 nuclease activity, the presence of the Bth mutation, and the presence of the sgRNA. (a) In Bth/t ears injected with Cas9:Tmc1-wt3:lipid, which targets the wild-type Tmc1 allele instead of the mutant Bth allele, ABR thresholds (blue) were comparable to or higher than those of uninjected controls (red) after 4 weeks. (b) Bth/+ ears injected with Cas9:GFP sgRNA:lipid (blue) did not show improved ABR thresholds 4 weeks after treatment. (c) Bth/+ears injected with catalytically inactive dCas9:Tmc1-mut1:lipid did not show improved ABR thresholds 4 weeks after treatment. (d) ABR thresholds of wild-type $\mathrm{C} 3 \mathrm{H}$ mice injected with Cas9:Tmc1-mut3:lipid showed similar patterns as the uninjected control inner ears at 4 weeks, except at 5.66 and $45.24 \mathrm{kHz}$ where ABR thresholds were elevated. (e) Elevated DPOAE thresholds at three frequencies were observed after the treatment in (d). (f) Injection of Cas9:Lipofectamine 2000 (LPF2000) without sgRNA in Bth/+ mice did not improve ABR thresholds after 4 weeks. (g) Elevated DPOAE thresholds at 11 and $16 \mathrm{kHz}$ were observed after the treatment in (f). Statistical analysis of ABR and DPOAE thresholds was performed by two-way ANOVA with Bonferroni correction for multiple comparisons: * $p<$ $0.05, * * p<0.01, * * * p<0.001$, and $* * * * p<0.0001$. Values and error bars reflect mean \pm SEM. Among the different frequencies assayed, the number of ears tested (n) varies within the range shown (see Supplementary Table 2). 
a

b

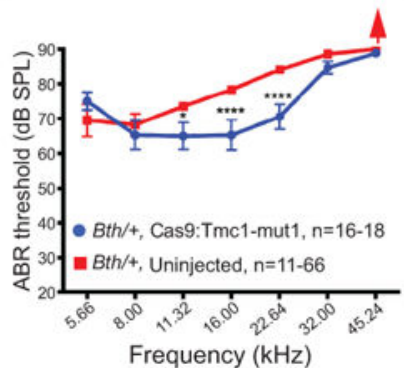

C
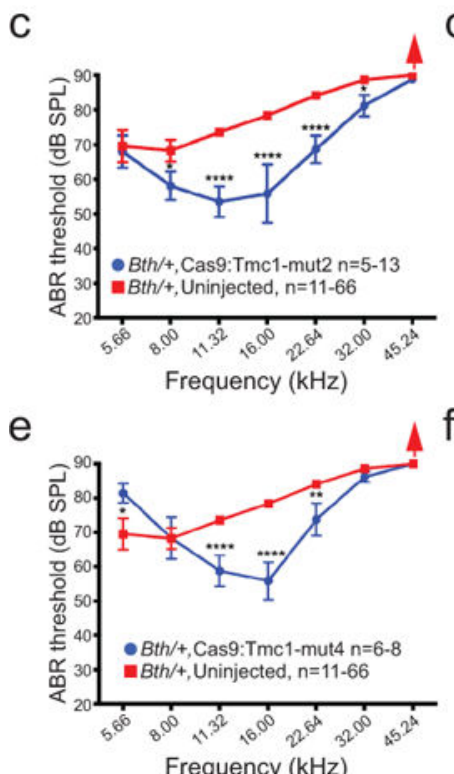

d

$f$
Bth/+Cas9:Tmc1-mut1, $\mathrm{n}=16-18$

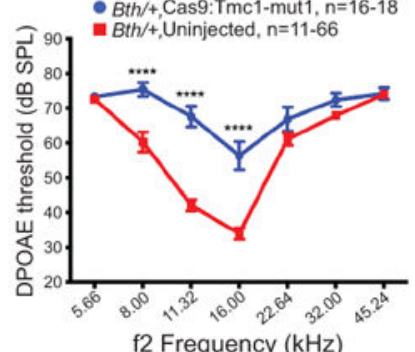

f2 Frequency $(\mathrm{kHz})$
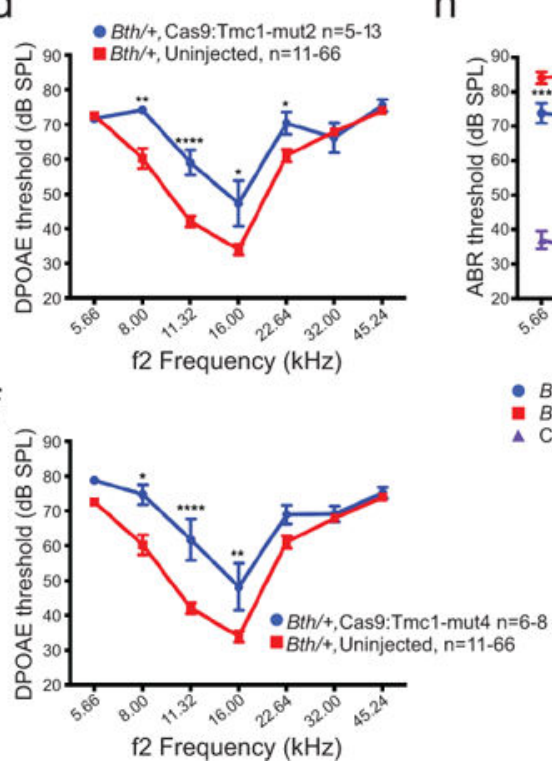

$\mathrm{h}$ g
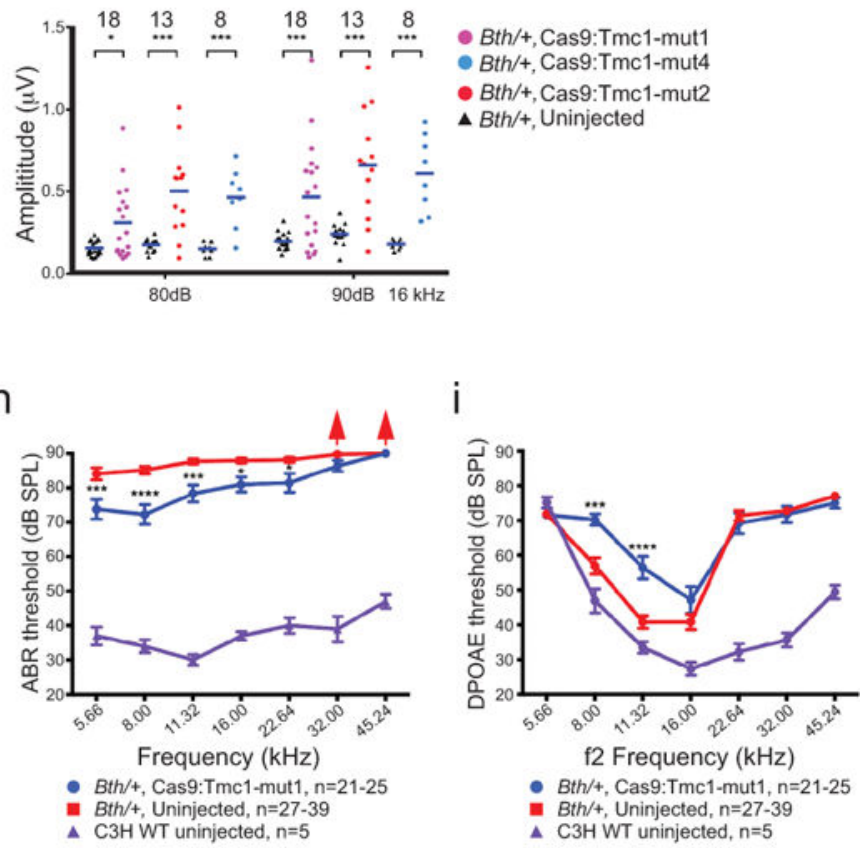

Extended Data Figure 7.

Hearing preservation following treatment with additional Tmc1-mut sgRNAs other than

Tmc1-mut3. (a) Mean ABR thresholds were significantly reduced at three frequencies in ears injected with Cas9:Tmc1-mut1:lipid compared to uninjected Bth/+ ears after 4 weeks.

(b) DPOAE thresholds were elevated in the same group of inner ears after Cas9:Tmc1-mut1 injection as in (a) after 4 weeks. (c) Mean ABR thresholds were significantly reduced at five frequencies in ears injected with Cas9:Tmc1-mut2:lipid compared to uninjected Bth/+ ears after 4 weeks. (d) DPOAE thresholds were elevated in the same group of inner ears after Cas9:Tmc1-mut2 injection as in (c) after 4 weeks. (e) Mean ABR thresholds were significantly reduced at three frequencies in ears injected with Cas9:Tmc1-mut4:lipid compared to uninjected $B t h /+$ ears after 4 weeks. (f) DPOAE thresholds were elevated in the same group of inner ears after Cas9:Tmc1-mut4:lipid injection as in (e) after 4 weeks. (g) Significantly stronger Wave 1 amplitudes were detected in ears injected with each of the Cas9:Tmc1-mut:lipid complexes shown at $16 \mathrm{kHz}$ ( 80 and $90 \mathrm{~dB}$ SPL). (h) 8 weeks after Cas9:Tmc1-mut1:lipid injection into Bth/+ ears, mean ABR thresholds were significantly reduced at five frequencies compared to the uninjected $B t h /+$ ears, which showed ABR thresholds $>80 \mathrm{~dB}$ at all frequencies after 8 weeks. Mean ABR thresholds of untreated wildtype (WT) $\mathrm{C} 3 \mathrm{H}$ mice of 8 weeks of age are shown in purple. Red arrows indicate no ABR response at the highest SPL level of $90 \mathrm{~dB}$. (i) DPOAE thresholds were modestly 
elevated in the same group of inner ears after Cas9:Tmc1-mut1 injection as in (h) after 8 weeks. Mean DPOAE thresholds of untreated wildtype (WT) $\mathrm{C} 3 \mathrm{H}$ mice of 8 weeks of age are shown in purple. Statistical analysis of ABR and DPOAE thresholds and Wave 1 amplitudes was performed by two-way ANOVA with Bonferroni correction for multiple comparisons: $* p<0.05, * * p<0.01, * * * p<0.001$, and $* * * * p<0.0001$. Values and error bars reflect mean \pm SEM. Among the different frequencies assayed, the number of ears tested (n) varies within the range shown (see Supplementary Table 2).

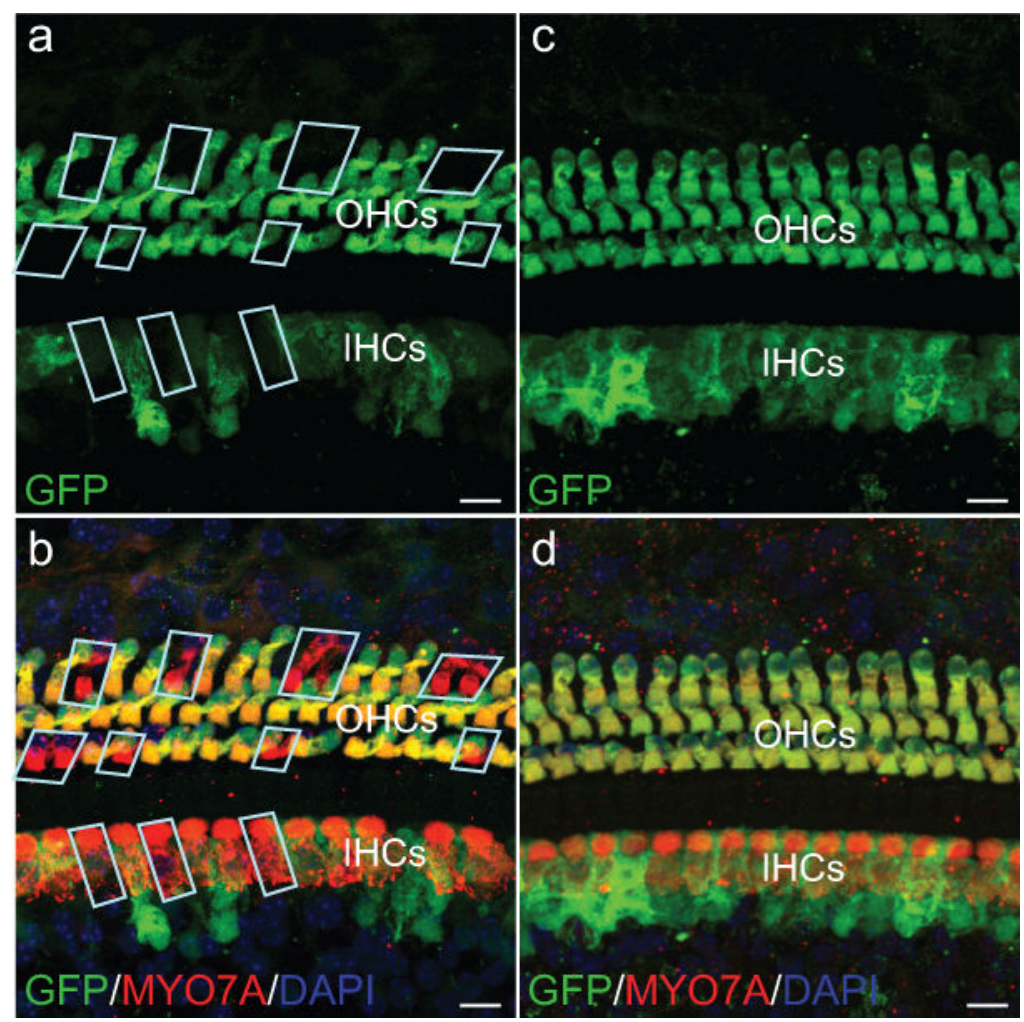

\section{Extended Data Figure 8.}

RNP delivery of Cas9:sgRNA:lipid complexes results in genome editing in adult hair cells. 6-week-old adult Atoh1-GFP cochlea were injected with $1 \mu \mathrm{L} 25 \mu \mathrm{M}$ Cas9:GFP sgRNA:lipid complex by canalostomy, with the cochlea harvested two weeks after injection. (a) Genome editing was detected by the loss of GFP (green, with GFP absence noted using cyan shapes) in inner hair cells (IHCs) and outer hair cells (OHCs) (b) Hair cells were labeled with a hair cell marker MYO7A (red) in the apex turn of cochlea. (c, d) In uninjected contralateral Atoh1-GFP cochlea, all hair cells were GFP-positive. Scale bars $=10 \mu \mathrm{m}$. Similar results were observed in other injected ears that were immunolabeled $(n=3)$. 

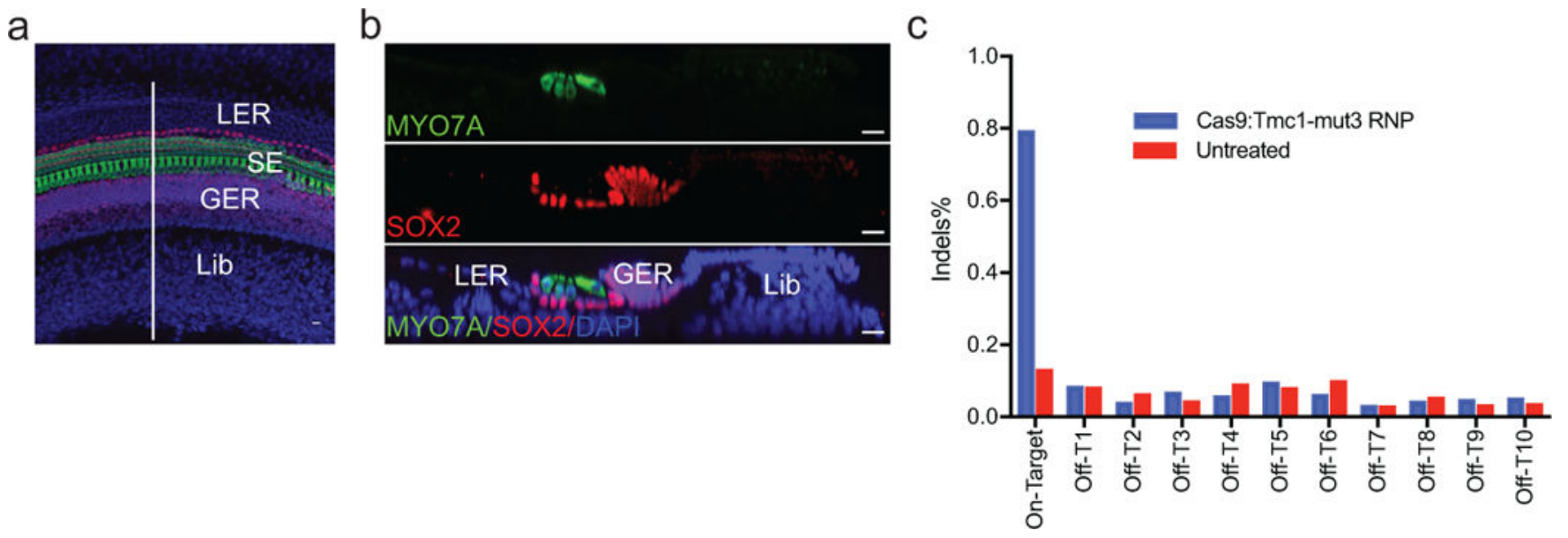

Extended Data Figure 9.

In vivo editing of the Tmc1 locus from Bth/+ ears injected with Cas9:Tmc1-mut3 sgRNA. A representation of the organ of Corti harvested at P5 for high-throughput DNA sequencing: (a) A confocal z-stack image showing the surface view of a dissected and labeled organ of Corti used for HTS. (b) A cross-sectional view of the organ of Corti (along the white line in (a)) showing the positions of hair cells (MYO7A), supporting cells (SOX2) and the cells from other cochlear regions that were used for quantification. LER: lesser epithelial ridge; GER: greater epithelial ridge; SE: sensory epithelium; Lib: The limbus region. DAPI-labeled nuclei are shown in blue. Quantification showed hair cells represented $1.45 \% \pm 0.05 \%$ (mean \pm SEM, $n=4$ ) of all the cells in the dissected cochlea. Scale bars $=10 \mu \mathrm{m}$. (c) Ontarget and off-target in vivo editing of the Tmc1 locus in organ of Corti samples. No indels were observed at frequencies substantially above that of an untreated control sample at any of the ten off-target sites identified by GUIDE-seq (Off-T1 to Off-T10). Indels were detected by HTS at the Tmc1 on-target site and each off-target site from in vivo tissue samples dissected from the inner ear of neonatal mouse 5 days after Cas9:Tmc1-mut3 RNP injection (blue), or from untreated control samples (red). 


\section{Extended Data Table 1}

Off-target editing after nucleofection of DNA plasmids encoding Cas9 and Tmc1-mut3 sgRNA into primary fibroblasts derived from Bth/Bth mice. (a) Off-target sties identified by GUIDE-seq ${ }^{25}$. Mismatch positions are indicated counting the PAM as positions 21-23. Off$T 3$, off- $T 6$, off- $T 7$, off- $T 8$ and off- $T 9$ are located within predicted gene regions, while the rest are intergenic. $1000 \mathrm{ng}$ Cas9 plasmid and $300 \mathrm{ng}$ Tmc1-mut3 sgRNA plasmid were nucleofected into Bth/Bth fibroblasts using a LONZA 4D-Nucleofector and indels were detected by HTS at Tmc1 Bth on-target and each off-target site. Mismatches compared to the on-target sequence are shown in red and PAMs are in blue; (b) Off-target sites identified by computational prediction using the CRISPR Design Tool ${ }^{24}$. Among the top eight computationally predicted off-target sites, only two (off- $T 1^{\prime}$ and off-T2 ${ }^{\prime}$ with two and three mismatches, respectively) were identified as bona fide off-targets in cells by GUIDE-seq. 1000 ng Cas9 plasmid and 300 ng Tmc1-mut3 sgRNA plasmid were nucleofected into $B$ th/Bth fibroblasts using a LONZA 4D-Nucleofector. Indels were detected by HTS at the Tmc1 Bth on-target site and at each predicted off-target site. Mismatches are shown in red and PAMs are in blue.

\begin{tabular}{|c|c|c|c|c|c|c|}
\hline \multicolumn{7}{|l|}{ a } \\
\hline & $5^{\prime}$-Sequence-3' & Mismatches (MMs) & NCBI accession & Predicted function & Location & Indels in Bth/Bth \\
\hline Bth & GGGTGGGACAGAACTTCCCCAGG & oMMs & N/A & & chr9 & $31 \%$ \\
\hline Off-TI & GGGAGGGACAGAGCTTCCCCAGG & 2 MMs [4:13] & N/A & & chr1 & $8.1 \%$ \\
\hline Off-T2 & GTGAGGGAGAGAACTTCCCCTGG & 3MMs [2:4:9] & N/A & & $\operatorname{chr} 16$ & $4.4 \%$ \\
\hline Off-T3 & AGTTGGTACAGAACTTCCCCAGG & 3MMs [1:3:7] & NC_000068.7 & CD82 antigen & chr2 & $2.6 \%$ \\
\hline Off-T4 & TTGTGGGACAGAAATTCCCCAGG & 3 MMs [1:2:14] & N/A & & chr12 & $3.9 \%$ \\
\hline Off-T5 & AGAGGAGACAGAACTCCCCCAGG & 5MMs [1:3:4:6:16] & N/A & & chr13 & $3.4 \%$ \\
\hline Off-T6 & GGGTGGGACAGATCTTCCCAGGG & 2MMs [13:20] & NC_000067.6 & hemicentin-1 isoform & chr1 & $0.68 \%$ \\
\hline Off- $-T 7$ & GTGTAGGACAGAACTTCGCCAGG & 3 MMs [2:5:18] & XM_006507026.3 & $\begin{array}{l}\text { inositol 1,4,5- } \\
\text { triphosphate receptor } \\
2\end{array}$ & chr6 & $1.5 \%$ \\
\hline Off-TS & GGTGAGACCAGAGCTTCCCCTGG & 6MMs [3:4:5:7:8:13] & XR_389309.3 & unknown & chr5 & $1.2 \%$ \\
\hline Off-T9 & AGGTGGGAAAGAACTTCTCCGGG & $3 \mathrm{MMs}[1: 9: 18]$ & NC_000070.6 & $\begin{array}{c}\text { paralemmin A kinase } \\
\text { anchor protein }\end{array}$ & chr4 & $1.4 \%$ \\
\hline Off-TIO & GGGTGGTAAAGAACTTCTCCTGG & 3MMs [7:9:18] & N/A & & $\operatorname{chr} 10$ & $0.048 \%$ \\
\hline
\end{tabular}

\begin{tabular}{|c|c|c|c|c|c|}
\hline \multicolumn{6}{|l|}{ b } \\
\hline & $5^{\prime}$-Sequence-3' & Mismatches & NCBI accession & Location & Indels in $B t h / B t h$ \\
\hline Bth & GGGTGGGACAGAACTTCCCCAGG & oMMs & & chr9 & $31 \%$ \\
\hline Off-TI' & GGGAGGGACAGAGCTTCCCCAGG & 2MMs [4:13] & & chr1 & $8.1 \%$ \\
\hline Off-T2' & GTGAGGGAGAGAACTTCCCCTGG & 3MMs [2:4:9] & & chr16 & $4.4 \%$ \\
\hline
\end{tabular}

Nature. Author manuscript; available in PMC 2018 June 20. 


\begin{tabular}{|c|c|c|c|c|c|}
\hline \multicolumn{6}{|l|}{ b } \\
\hline & $5^{\prime}$-Sequence-3' & Mismatches & NCBI accession & Location & Indels in $B$ th/Bth \\
\hline Off-T3' & AGGAAGGCCAGAACTTCCCCTAG & 4MMs [1:4:5:8] & NM_001312644.1 & chr12 & $0.037 \%$ \\
\hline Off-T4' & GGAGGGGGCTGAACTTCCCCAGG & 4MMs [3:4:8:10] & & chr9 & $0.071 \%$ \\
\hline Off-TS & GTGTGGAACAGAACTTCCCAGGG & 3MMs [2:7:20] & & chrl & $0.046 \%$ \\
\hline Off-T6 & CCCTGGAACAGAACTTCCCCAAG & 4MMs [1:2:3:7] & & chr2 & $0.097 \%$ \\
\hline Off- $T 7^{\prime}$ & GCGCGGGACAGAACATCCCCTAG & 3MMs [2:4:15] & & chr5 & $0.033 \%$ \\
\hline Off-T $T 8^{\prime}$ & AGGCAGGACAAAACTTCCCCAAG & 4MMs [1:4:5:11] & & chr1 & $0.091 \%$ \\
\hline
\end{tabular}

\section{Supplementary Material}

Refer to Web version on PubMed Central for supplementary material.

\section{Acknowledgments}

This work was supported by DARPA HR0011-17-2-0049 (to D.R.L.), and US NIH R01 EB022376 (to D.R.L.), R35 GM1 18062 (to D.R.L.), R01 DC006908 (to Z.Y.C), P30 DC05209 (to M.C.L.), R01 DC00138 (to M.C.L.), and R01 DC013521 (to J.R.H.). We are grateful for support from the David-Shulsky Foundation (to Z.Y.C.), a Frederick and Ines Yeatts Hair Cell Regeneration grant (to Y.T., V.L., M.H., and Y.S.), the Bertarelli Foundation (to J.R.H.), the Broad Institute (to D.R.L and Z.Y.C.), and HHMI (to D.R.L.). We thank Holly Rees, Shengdar Tsai, Michael Packer, and Dmitry Usanov, for assistance.

\section{References}

1. Angeli S, Lin X, Liu XZ. Genetics of hearing and deafness. Anat Rec (Hoboken). 2012; 295:18121829. [PubMed: 23044516]

2. Marazita ML, et al. Genetic epidemiological studies of early-onset deafness in the U.S. school-age population. Am J Med Genet. 1993; 46:486-491. [PubMed: 8322805]

3. Morton CC, Nance WE. Newborn hearing screening-a silent revolution. N Engl J Med. 2006; 354:2151-2164. [PubMed: 16707752]

4. Geleoc GS, Holt JR. Sound strategies for hearing restoration. Science. 2014; 344:1241062. [PubMed: 24812404]

5. Muller U, Barr-Gillespie PG. New treatment options for hearing loss. Nat Rev Drug Discov. 2015; 14:346-365. [PubMed: 25792261]

6. Shibata SB, et al. RNA Interference Prevents Autosomal-Dominant Hearing Loss. Am J Hum Genet. 2016; 98:1101-1113. [PubMed: 27236922]

7. Pan B, et al. Gene therapy restores auditory and vestibular function in a mouse model of Usher syndrome type 1c. Nat Biotechnol. 2017

8. Sun H, Huang A, Cao S. Current status and prospects of gene therapy for the inner ear. Hum Gene Ther. 2011; 22:1311-1322. [PubMed: 21338273]

9. Sacheli R, Delacroix L, Vandenackerveken P, Nguyen L, Malgrange B. Gene transfer in inner ear cells: a challenging race. Gene Ther. 2013; 20:237-247. [PubMed: 22739386]

10. Komor AC, Badran AH, Liu DR. CRISPR-Based Technologies for the Manipulation of Eukaryotic Genomes. Cell. 2017; 168:20-36. [PubMed: 27866654]

11. Long C, et al. Postnatal genome editing partially restores dystrophin expression in a mouse model of muscular dystrophy. Science. 2016; 351:400-403. [PubMed: 26721683]

12. Yang Y, et al. A dual AAV system enables the Cas9-mediated correction of a metabolic liver disease in newborn mice. Nat Biotechnol. 2016; 34:334-338. [PubMed: 26829317] 
13. Yin $\mathrm{H}$, et al. Therapeutic genome editing by combined viral and non-viral delivery of CRISPR system components in vivo. Nat Biotechnol. 2016; 34:328-333. [PubMed: 26829318]

14. Kim S, Kim D, Cho SW, Kim J, Kim JS. Highly efficient RNA-guided genome editing in human cells via delivery of purified Cas9 ribonucleoproteins. Genome Res. 2014; 24:1012-1019. [PubMed: 24696461]

15. Zuris JA, et al. Cationic lipid-mediated delivery of proteins enables efficient protein-based genome editing in vitro and in vivo. Nat Biotechnol. 2015; 33:73-80. [PubMed: 25357182]

16. Pan B, et al. TMC1 and TMC2 are components of the mechanotransduction channel in hair cells of the mammalian inner ear. Neuron. 2013; 79:504-515. [PubMed: 23871232]

17. Camp, GV., Smith, R. Hereditary Hearing loss Homepage. 2017. http://hereditaryhearingloss.org

18. Zhao Y, et al. A novel DFNA36 mutation in TMC1 orthologous to the Beethoven (Bth) mouse associated with autosomal dominant hearing loss in a Chinese family. PLoS One. 2014; 9:e97064. [PubMed: 24827932]

19. Kurima K, et al. Dominant and recessive deafness caused by mutations of a novel gene, TMC1, required for cochlear hair-cell function. Nat Genet. 2002; 30:277-284. [PubMed: 11850618]

20. Kawashima Y, Kurima K, Pan B, Griffith AJ, Holt JR. Transmembrane channel-like (TMC) genes are required for auditory and vestibular mechanosensation. Pflugers Arch. 2015; 467:85-94. [PubMed: 25074487]

21. Vreugde S, et al. Beethoven, a mouse model for dominant, progressive hearing loss DFNA36. Nat Genet. 2002; 30:257-258. [PubMed: 11850623]

22. Fu Y, Sander JD, Reyon D, Cascio VM, Joung JK. Improving CRISPR-Cas nuclease specificity using truncated guide RNAs. Nat Biotechnol. 2014; 32:279-284. [PubMed: 24463574]

23. Tsai SQ, et al. GUIDE-seq enables genome-wide profiling of off-target cleavage by CRISPR-Cas nucleases. Nat Biotechnol. 2015; 33:187-197. [PubMed: 25513782]

24. Zhang, F. CRISPR DESIGN. 2015. http://crispr.mit.edu

25. Lenz JJ, Jodelka FM, et al. Rescue of hearing and vestibular function by antisense oligonucleotides in a mouse model of human deafness. Nat Med. 2013; 19:345-50. [PubMed: 23380860]

26. Wang M, et al. Efficient Delivery of Genome-Editing Proteins using Bioreducible Lipid Nanoparticles. Proc Natl Acad Sci. 2015; 113:2868-2873.

27. Pattanayak V, et al. High-throughput profiling of off-target DNA cleavage reveals RNAprogrammed Cas9 nuclease specificity. Nat Biotechnol. 2013; 31:839-843. [PubMed: 23934178]

28. Lumpkin EA, et al. Math1-driven GFP expression in the developing nervous system of transgenic mice. Gene Expr Patterns. 2003; 3:389-395. [PubMed: 12915300]

29. Huang M, et al. Hair cell overexpression of Islet1 reduces age-related and noise-induced hearing loss. J Neurosci. 2013; 33:15086-94. [PubMed: 24048839] 
a Targeted region of the Tmc1 Bth allele 5'- TGTCCCTCCTGGGGAAGTTCTGTCCCACCCTGT -3' 3'- ACAGGGAGGACCCCTTCAAGACAGGGTGGGACA -5' Tmc1-mut1 Tmc1-mut2 Tmc1-mut3 Tmc1-mut4

Protospacer PAM

Tmc1-mut1: 5'- GGGACAGAACTTCCCCAGGAGGG -3'

Tmc1-mut2: 5'- TGGGACAGAACTTCCCCAGGAGG -3'

Tmc1-mut3: 5'- GGGTGGGACAGAACTTCCCCAGG -3'

Tmc1-mut4: 5'- TGGGACAGAACTTCCCCAGG -3'

b

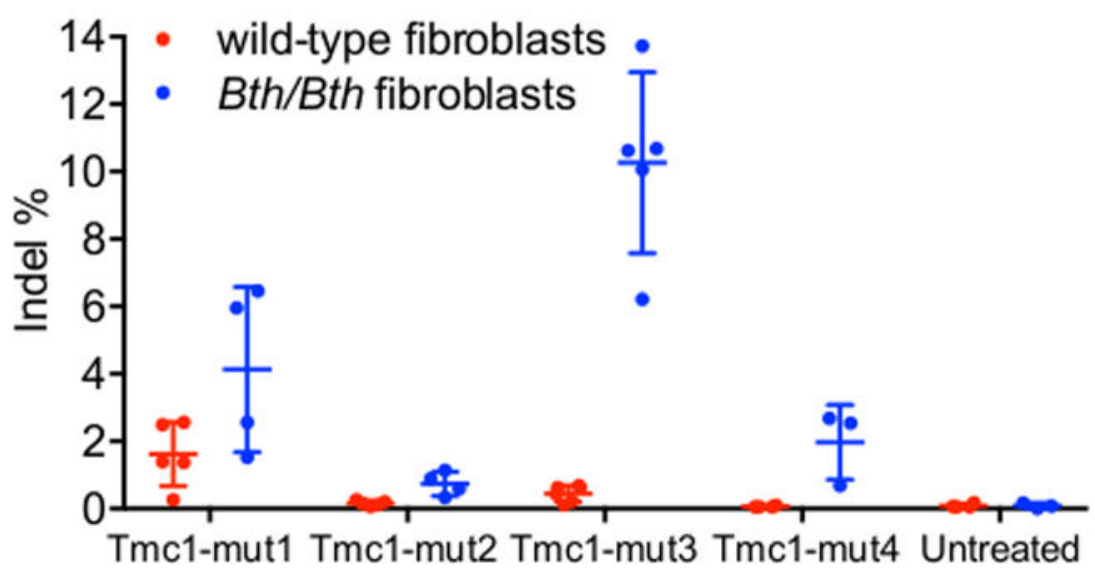

Figure 1. Design of a genome-editing strategy to disrupt the Bth mutant allele

(a) SpCas9 $\operatorname{sgRNAs}$ were designed to target the mutant Tmc1 Bth allele, in which T1235 is changed to A (red). The protospacer (blue arrows) of each Bth-targeting sgRNA contains a complementary $\mathrm{T}$ (red) that pairs with the T1235A mutation in the Bth allele, but that forms a mismatch with wild-type Tmc1 allele. (b) Lipid-mediated delivery of Cas9:sgRNA complexes into primary fibroblasts derived from wild-type or homozygous Bth/Bth mice. $100 \mathrm{nM}$ of purified Cas9 protein and $100 \mathrm{nM}$ of each sgRNA shown were delivered using Lipofectamine 2000. Indels were quantitated by HTS. Values and error bars represent the mean \pm SD of three or more independent biological replicates. 
a

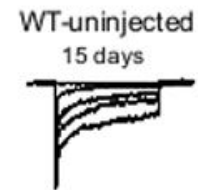

21 days

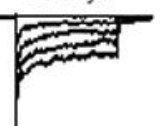

C

f

b
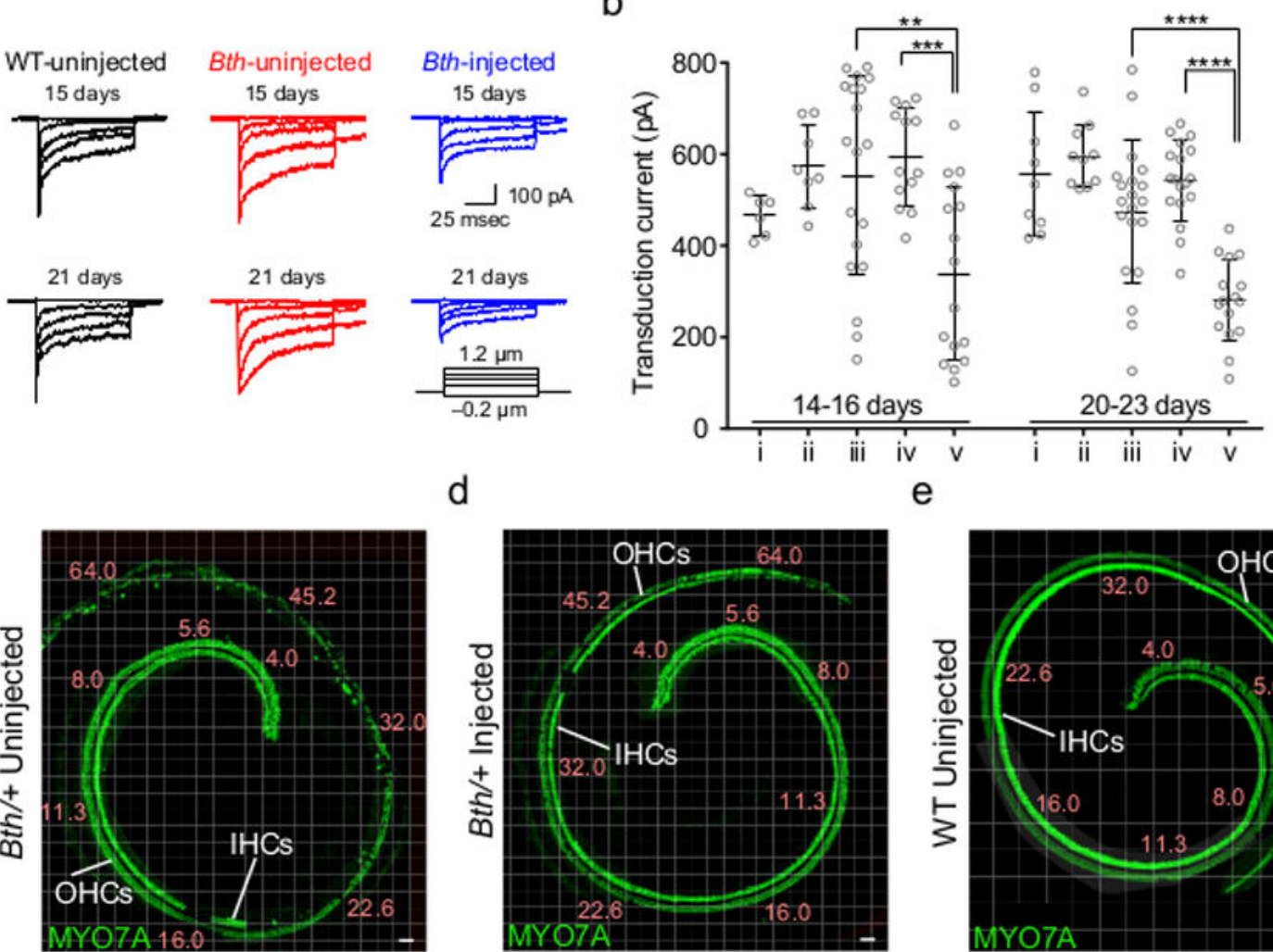

d
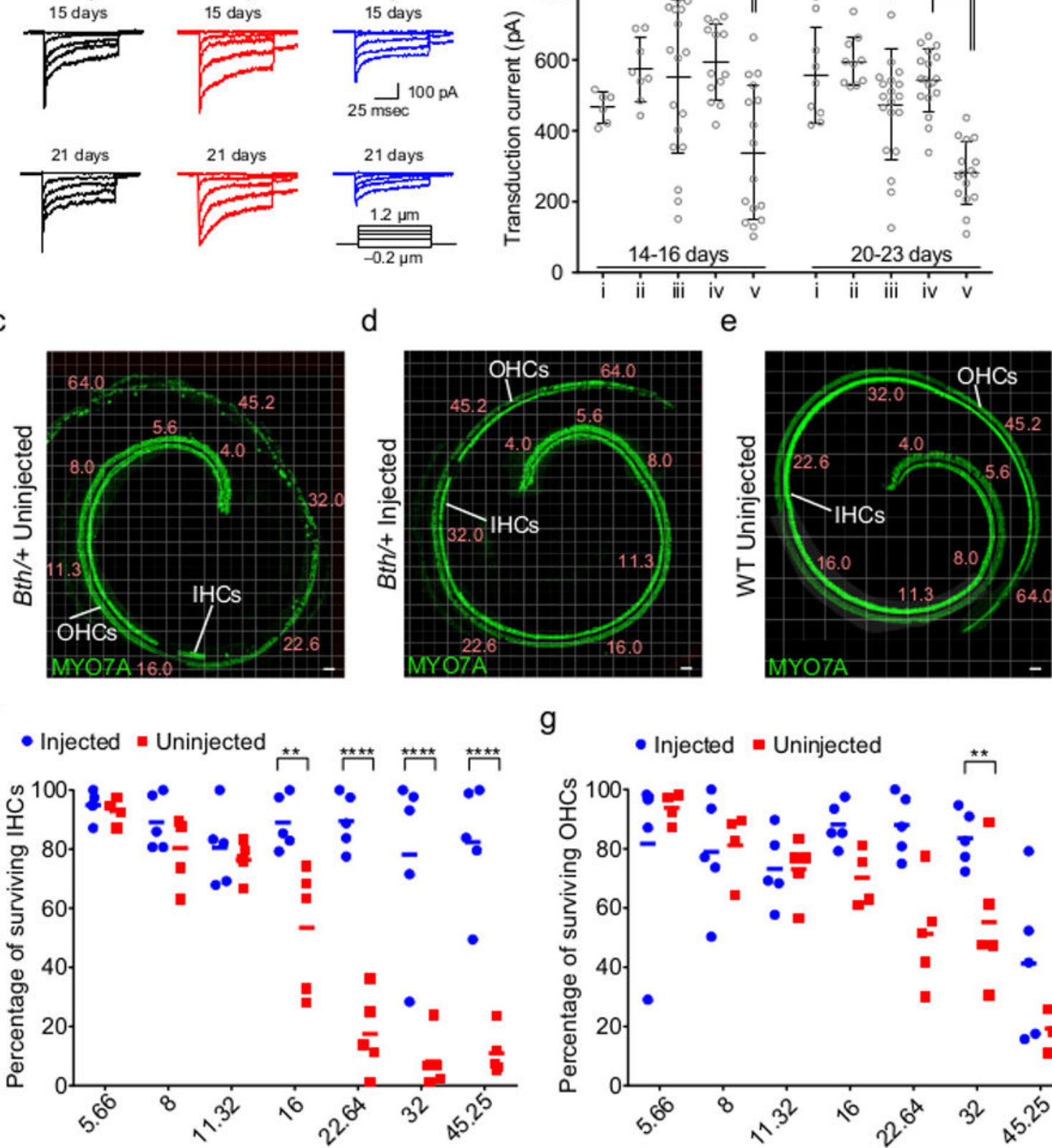

g

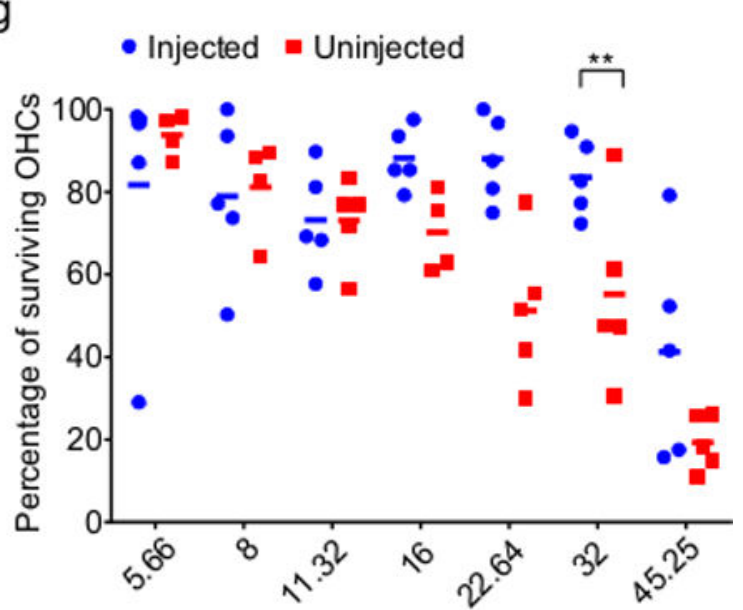

Cochlear regions corresponding to frequency $(\mathrm{kHz})$

Figure 2. Effects of Cas9:Tmc1-mut3 sgRNA:lipid injection on hair-cell function and hair-cell survival in mice

(a) Representative transduction currents from inner hair cells (IHCs) of P0-P1 wild-type or Tmc1 Bth/ $\Delta ;$ Tmc2 $/ \Delta$ mice that were uninjected, or injected with Cas9:Tmc1-mut3:lipid complex. (b) Maximal transduction current amplitudes for 135 IHCs from P1 wild-type C57B/L6 and Tmc1 Bth/A;Tmc24/4 mice. (i): uninjected wild-type C57B/L6 mice; (ii): wild-type C57B/L6 mice injected with Cas9:Tmc1-wt3:lipid; (iii): uninjected Tmc1 Bth/ $\Delta ; T m c 2 \Delta \Delta$ mice; (iv): Tmc1 Bth/ $\Delta ; T m c 2 \Delta \Delta$ mice injected with Cas9:GFP-targeting

Nature. Author manuscript; available in PMC 2018 June 20. 
sgRNA:lipid; (v): Tmc1 Bth/4;Tmc2 $\Delta / \Delta$ mice injected with Cas9:Tmc1-mut3:lipid.

Horizontal lines and error bars reflect mean \pm SD. (c-e) Representative confocal microscopy images from (c) an uninjected Bth/+ cochlea; (d) the contralateral cochlea of the mouse in (c) injected with Cas9:Tmc1-mut3:lipid complex at P1; (e) an untreated wild-type $\mathrm{C} 3 \mathrm{H}$ cochlea. Numbers in pink indicate approximate frequencies (in $\mathrm{kHz}$ ) sensed by each region. Scale bars $=50 \mu \mathrm{m}$. (f, g) Quantification of IHC (f) and OHC (g) survival in Bth/+ mice relative to wild-type $\mathrm{C} 3 \mathrm{H}$ mice 8 weeks after Cas9:Tmc1-mut3:lipid injection (blue) compared to uninjected (red) contralateral ears. Values and error bars reflect the means and SEMs of five biological replicates. Statistical tests in (b) are two-population T-tests, and in (f) and (g) are two-way ANOVA with Bonferroni correction: ${ }^{* *} p<0.01,{ }^{* * *} p<0.001$, and $* * * * p<0.0001$. 
a

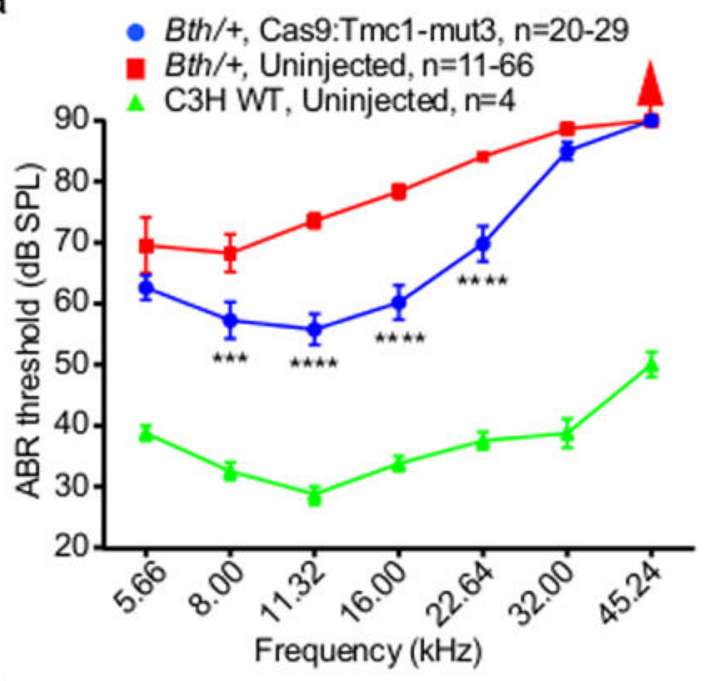

C

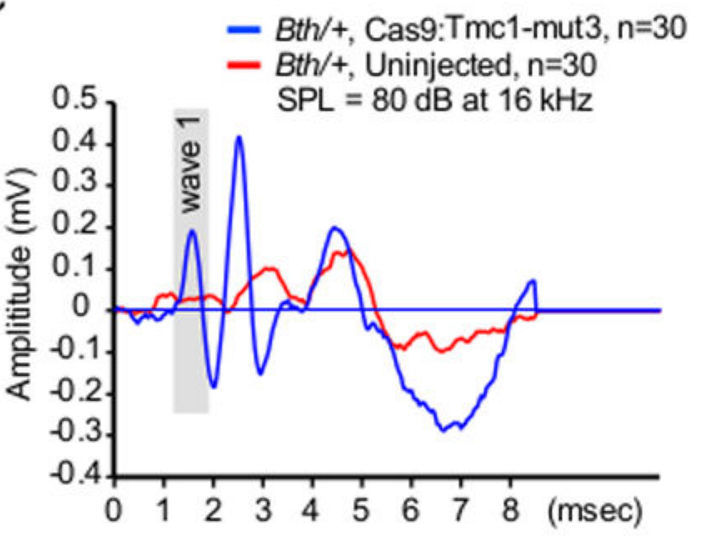

b

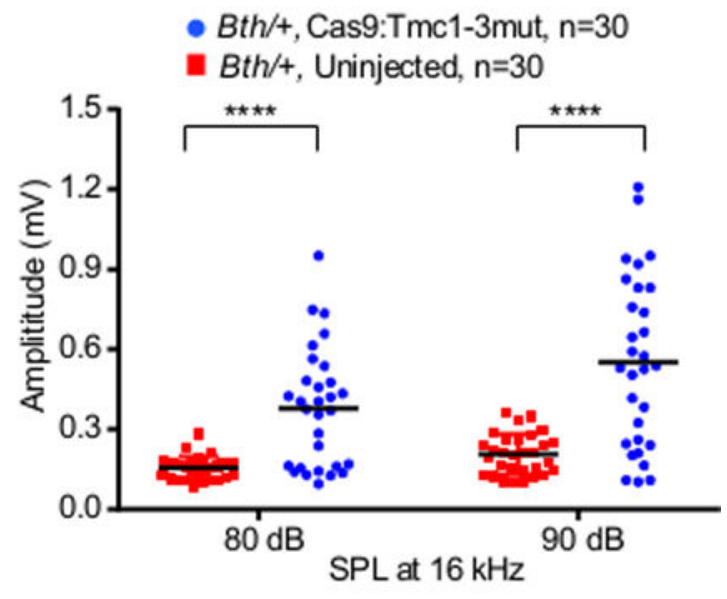

d

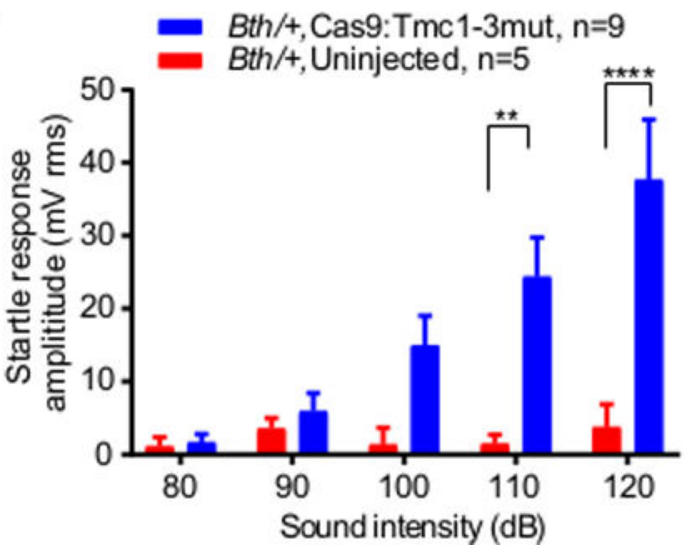

Figure 3. Cas9:Tmc1-mut3 sgRNA:lipid injections reduce hearing loss in $B t h /+$ mice (a) ABR thresholds in Bth/+ ears injected with Cas9:Tmc1-mut3:lipid (blue), uninjected Bth/+ ears (red), and wild-type $\mathrm{C} 3 \mathrm{H}$ ears (green) after 4 weeks. (b) Amplitudes of ABR Wave 1 at $16 \mathrm{kHz}$ in Cas9:Tmc1-mut3:lipid-injected ears (blue) compared with uninjected ears (red) after 4 weeks. Horizontal bars are mean values. (c) Mean ABR waveforms in Cas9:Tmc1-mut3:lipid-injected ears (blue) and uninjected ears (red). (d) Startle responses in Cas9:Tmc1-mut3:lipid-injected mice (blue) and in uninjected mice (red) 8 weeks after treatment. Red arrows in (a) indicate no ABR response at the highest stimulus level tested $(90 \mathrm{~dB})$. See also Supplementary Table 2. Statistical tests were two-way ANOVA with Bonferroni correction for multiple comparisons: $* * p<0.01$, ***p $p<0.001$, and $* * * p<$ 0.0001. Values and error bars reflect mean \pm SEM. 
a

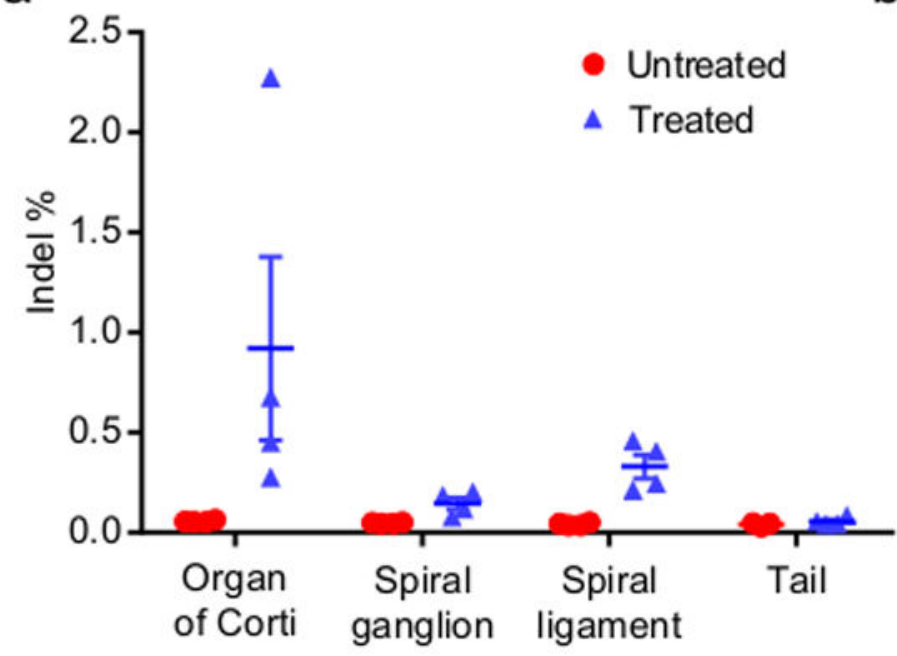

b

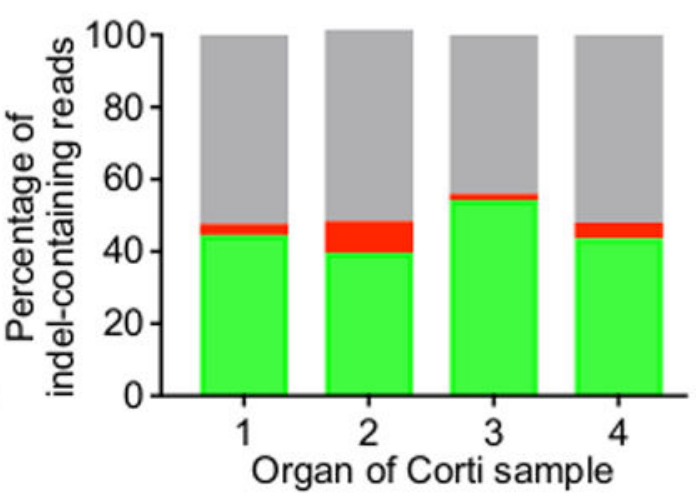

C

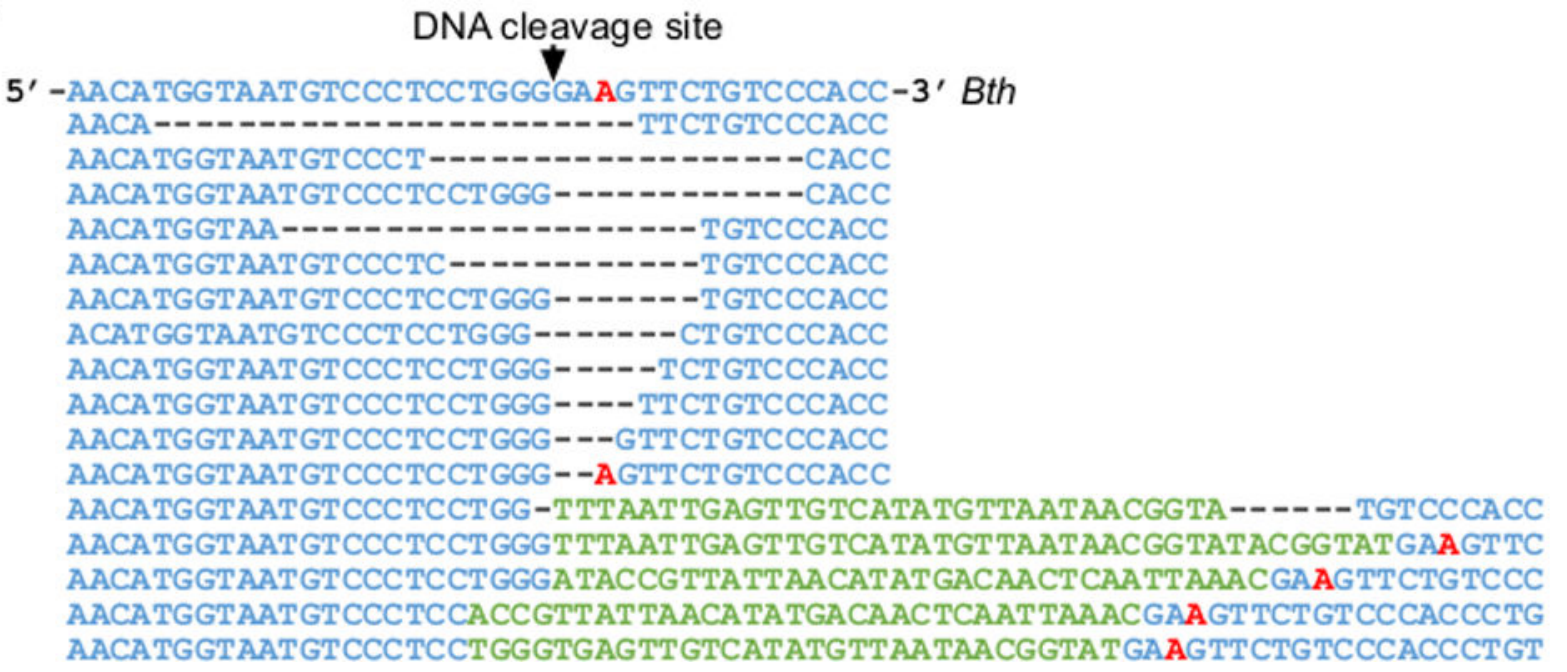

Figure 4. Genome modification at Tmc1 induced by lipid-mediated delivery of Cas9:Tmc1-mut3 RNP into $B$ th/+ mice

(a) Tmc1 indel frequencies from tissue samples 5 days after injection of Cas9:Tmc1mut3:lipid (blue) or from uninjected mice (red). Horizontal lines and error bars reflect mean \pm SEM. Note that $B t h$ allele indel frequencies in these heterozygous mice are approximately double the observed indel frequencies. (b) Analysis of indel-containing Tmc1 sequencing reads from four injected organ of Corti samples in (a). (c) The most abundant 16 Tmc1 sequences, grouped by similarity, from organ of Corti samples in (b). The T1235A Bth mutation is red. 